\title{
Long-time asymptotics of the second grade fluid equations on $\mathbb{R}^{2}$
}

\author{
Basma Jaffal-Mourtada
}

Communicated by Y. Charles Li, received May 25, 2011.

\begin{abstract}
We study the large time behavior of solutions of the second grade fluid system in the space $\mathbb{R}^{2}$. Using scaled variables and introducing several functionals in weighted Sobolev spaces, we prove that the solution of the second grade fluid equations converges to the Oseen vortex, if the initial data are small enough. We also give an estimate of the rate of convergence.
\end{abstract}

\section{Contents}

1. Introduction 185

2. Preliminaries and regularized vorticity equation 190

3. Spectral study of the operator $\mathcal{L}$ and decomposition of the solution $\quad 195$

4. Asymptotic behavior of solutions and energy estimates 200

5. Proof of Theorem 3.3 and convergence when $\epsilon$ tends to zero 212

References

\section{Introduction}

The classical theory of Newtonian fluids is unable to explain properties observed in some fluids in the nature. Most of such fluids belong to the class of non-Newtonian fluids. This is the case, for example, of many polymer solutions and many commonly substances found in the industry (petroleum industry, plastic manufacture, application of paints,....).

Several models have been introduced to describe and explain the behavior of nonNewtonian fluids. Among these models, fluids of differential type introduced by Rivlin-Erickson [23] have attracted much attention from a theoretical point of view. In this article, we are interested in the study of a special class of non-Newtonian fluids of differential type, namely fluids of second grade. Their study was initiated in

1991 Mathematics Subject Classification. Primary 35; Secondary 76.

Key words and phrases. Second grade fluid system, weighted Sobolev spaces, Oseen vortex. 
1974 by J.E.Dunn and R.L.Fosdick [7] and then by R.L.Fosdick and K.R.Rajakopal [8], [9]. For such fluids, the Cauchy stress tensor $T$ is a polynomial of degree less than 2 in the first two Rivlin-Ericksen kinematical tensors $A_{1}$ and $A_{2}$.

$$
T=-p I+\nu A_{1}+\alpha\left(A_{2}-A_{1}^{2}\right)
$$

where $p$ is the pressure, $\nu$ is the viscosity and the tensors $A_{1}$ and $A_{2}$ are defined by

$$
\begin{gathered}
A_{1}=(\operatorname{grad} u)+(\operatorname{grad} u)^{T}, \\
A_{2}=\frac{d A_{1}}{d t}+A_{1}(\operatorname{grad} u)+(\operatorname{grad} u)^{T} A_{1}
\end{gathered}
$$

where $u$ is the velocity of the fluid and $\frac{d}{d t}=\partial_{t}+u$. $\nabla$.

The Newton laws and a classical computation lead to the following system, which describes the motion of an incompressible fluid of second grade

$$
(S G F)\left\{\begin{aligned}
\partial_{t}(u-\alpha \Delta u)+\operatorname{rot}(u-\alpha \Delta u) \times u & =\nu \Delta u-\nabla p \\
\operatorname{div} u & =0 \\
u(x, 0) & =u_{0}
\end{aligned}\right.
$$

where $u=u(x, t) \in \mathbb{R}^{2}$ is the velocity field, $p=p(x, t)$ is the pressure, $\alpha$ is a material coefficient, $\nu$ is the viscosity and $x \in \mathbb{R}^{2}, t \geq 0$.

In the system (SGF), we have used the following notations and identifications. We have identified any two-component vector field $v=\left(v_{1}, v_{2}\right)^{t}$ with the threecomponent field $v=\left(v_{1}, v_{2}, 0\right)^{t}$ and denoted rot $v$ the 3 -component vector field given by

$$
\operatorname{rot} v=\left(0,0, \partial_{1} v_{2}-\partial_{2} v_{1}\right)^{t}
$$

Several authors have been interested in the study of the second grade fluid equations $([\mathbf{1}],[\mathbf{6}],[\mathbf{1 0}],[\mathbf{1 1}],[\mathbf{1 2}],[\mathbf{1 8}],[\mathbf{2 1}],[\mathbf{2 0}])$. The first mathematical result of existence and uniqueness of solutions was obtained by Cioranescu and Ouazar in [6]. More precisely, when $\Omega$ is a bounded domain in $\mathbb{R}^{2}$, Cioranescu and Ouazar have proved that for divergence-free initial data $u_{0}$ in $\left(H^{3}(\Omega)\right)^{2} \cap\left(H_{0}^{1}(\Omega)\right)^{2}$, the solution of the system $(S G F)$ exists and is unique in the space $L^{\infty}\left(\mathbb{R}^{+},\left(H^{3}(\Omega)\right)^{2}\right) \cap$ $L^{2}\left(\mathbb{R}^{+},\left(H^{1}(\Omega)\right)^{2}\right)$. The existence and uniqueness results are the same in the case of periodic conditions, for more details, see $[\mathbf{2 1}]$. When the equations are considered in the whole space, a similar result is proved $[\mathbf{1}]$.

In the three-dimensional case, Cioranescu and Ouazar have also proved local existence (and uniqueness) of solutions of System $(S G F)$ (see [6]). Later, in [5], Cioranescu and Girault established global existence (and uniqueness) of solutions for small initial data.

On the other hand, the problem of existence of classical solutions has been studied in [11] by Galdi, Grobbelaar and Sauer, who showed local existence and uniqueness of classical solutions for $(S G F)$. Furthermore, when the size of the initial data is suitably restricted and when the coefficient $\alpha \rho$ is sufficiently large, where $\rho$ is the density of the fluid, they obtained a global existence result. Later, Galdi and Sequeira have relaxed the condition on $\alpha$ in $[\mathbf{1 2}]$.

Let us remark that, when $\alpha$ vanishes, we recover the classical system of the NavierStokes equations.

In this paper, we are interested in studying the large time asymptotic behavior of the solution of $(S G F)$ in the whole space $\mathbb{R}^{2}$. Our motivation comes from the 
case $\alpha=0$ (the Navier-Stokes equations). In [16], Gallay and Wayne used ideas from the theory of dynamical systems in order to determine the long-time behavior of solutions of the Navier-Stokes equations on $\mathbb{R}^{2}$. They showed that small solutions of the corresponding vorticity equation, with non-zero total vorticity, asymptotically approach the Oseen vortex. In their work, they constructed finite-dimensional invariant manifolds of these equations, and proved that all solutions in a neighborhood of the origin approach one of these manifolds with a rate which can be determined. Thus, computing the asymptotics of solutions is reduced to the task of determining the asymptotics of the resulting systems of ordinary differential equations on these invariant manifolds.

This result was improved later in $[\mathbf{1 7}]$, where the authors showed that the Oseen vortices are not only locally stable but also globally stable. In other words, any solution of the two-dimensional vorticity equation whose initial vorticity is integrable will approach one of the Oseen vortices. The proof of the global stability was based on the construction of a pair of Lyapunov functionals for the rescaled vorticity equation. Later, in [15], Gallay and Rodrigues gave an estimation of the time that the solutions of the two-dimensional vorticity equation take to reach a neighborhood of the Oseen vortex, when the initial data are integrable and well localized in space (in [24], Rodrigues extended these results to slightly inhomogeneous incompressible fluids).

In this paper, we will prove that the solutions of the system $(S G F)$ have the same behavior as the solutions of the Navier-Stokes equations, that is, the vorticities converge to an Oseen vortex when the time goes to infinity.

As in the case of the Navier-Stokes equations, we will determine the asymptotics of the solutions of the system $(S G F)$ by studying the evolution of the vorticity, rather than the velocity. This is especially convenient in the two-dimensional case, where the vorticity is a scalar.

In fact, taking the curl of the first equation in $(S G F)$, and using the identity

$$
\operatorname{rot}(\operatorname{rot} \tilde{u} \times u)=u \cdot \nabla(\operatorname{rot} \tilde{u}),
$$

which is true for any divergence free smooth vector fields $u$ and $\tilde{u}$ in $\mathbb{R}^{2}$, we obtain the following equation for the vorticity $w=\operatorname{rot} u$

$$
\partial_{t}(w-\alpha \Delta w)-\nu \Delta w+u \cdot \nabla(w-\alpha \Delta w)=0
$$

One can then recover the solution $u(x, t)$ of $(S G F)$ via the Biot-Savart law (see Section 2.1).

In order to understand the long-time asymptotics of $(S G F)$, it is helpful to introduce scaling variables. Scaling variables have been used in the study of the long-time behavior of parabolic and also damped hyperbolic equations, in particular to prove convergence to self-similar solutions (see for example $[\mathbf{1 6}],[\mathbf{1 7}],[\mathbf{1 3}]$ ) . Following the ideas of Gallay and Wayne in [16] (see also [13]), for any fixed, large enough time $T$, we introduce the new scaled variables

$$
\xi=\frac{x}{\sqrt{\nu(t+T)}}, \tau=\log (t+T)
$$

We also define the new functions $W(\xi, \tau)$ and $V(\xi, \tau)$ by

$$
w(x, t)=\frac{1}{t+T} W\left(\frac{x}{\sqrt{\nu(t+T)}}, \log (t+T)\right)
$$


and

$$
u(x, t)=\sqrt{\frac{\nu}{t+T}} V\left(\frac{x}{\sqrt{\nu(t+T)}}, \log (t+T)\right)
$$

where $w(x, t)$ is a solution of (1.1) and $u(x, t)$ is the corresponding velocity field. Let

$$
\tau_{0}=\log T, \bar{\alpha}=\frac{\alpha}{\nu T}, \bar{\epsilon}=\frac{\epsilon}{\nu^{2} T} .
$$

Then, $W(\xi, \tau)$ satisfies the following system

$$
\partial_{\tau}\left(W-\bar{\alpha} e^{-\tau} \Delta_{\xi} W\right)-\mathcal{L} W+V \cdot \nabla_{\xi}\left(W-\bar{\alpha} e^{-\tau} \Delta_{\xi} W\right)
$$

$$
+\bar{\alpha} e^{-\tau} \Delta_{\xi} W+\frac{\bar{\alpha}}{2} e^{-\tau} \xi \cdot \nabla_{\xi} \Delta_{\xi} W=0,
$$

where $W\left(\xi, \tau_{0}\right)=W_{0}(\xi)=T w_{0}(x)$ and

$$
\mathcal{L} W=\Delta W+\frac{1}{2} \xi \cdot \nabla W+W .
$$

The second idea that helps to understand the long-time asymptotics of $(S G F)$ is the introduction of weighted Sobolev spaces. For any $m \geq 0$, we define the Hilbert space $L^{2}(m)$ by

$$
L^{2}(m)=\left\{f \in L^{2}\left(\mathbb{R}^{2}\right) \quad / \quad \int_{\mathbb{R}^{2}}\left(1+|\xi|^{2}\right)^{m}|f|^{2} d \xi<\infty\right\}
$$

We denote

$$
\|f\|_{L^{2}(m)}=\left(\int_{\mathbb{R}^{2}}\left(1+|\xi|^{2}\right)^{m}|f|^{2} d \xi\right)^{\frac{1}{2}}<\infty .
$$

We notice that the spectrum of the operator $\mathcal{L}$ acting on $L^{2}(m)$ consists of a discrete spectrum and a continuous one. Choosing $m$ large enough, we can move the continuous spectrum to the left as much as wanted. The eigenvectors corresponding to the isolated eigenvalues can be computed explicitly and are rapidly decaying at infinity (For more details, see section 3.1).

If $m>1, L^{2}(m)$ is embedded into $L^{1}\left(\mathbb{R}^{2}\right)$. We denote by $L_{0}^{2}(m)$ the closed subspace of $L^{2}(m)$ given by

$$
L_{0}^{2}(m)=\left\{f \in L^{2}(m) / \int_{\mathbb{R}^{2}} f(\xi) d \xi=0\right\}
$$

We also define the higher order Sobolev spaces

$$
\begin{aligned}
& H^{1}(m)=\left\{f \in L^{2}(m) / \partial_{i} f \in L^{2}(m), i=1,2\right\} \\
& H^{2}(m)=\left\{f \in H^{1}(m) / \partial_{i} f \in H^{1}(m), i=1,2\right\}
\end{aligned}
$$

We also use the classical Lebesgue spaces $L^{p}\left(\mathbb{R}^{2}\right)$ equipped with the classical norm

$$
\|u\|_{L^{p}}=\left(\int_{\mathbb{R}^{2}}|u(x)|^{p} d x\right)^{\frac{1}{p}}, \text { for all } p \geq 1 .
$$

The first step in the study of the asymptotic behavior of the solutions of Equation (1.1) is to prove a local existence theorem in the weighted Sobolev spaces $H^{2}(m), m \geq 0$.

For this purpose and for some technical reasons, we regularize Equation (1.1) by adding the smoothing term $\epsilon \Delta^{2} w$. Then, we study the asymptotic behavior of the solution $w_{\epsilon}$ of the regularized equation and establish energy estimates that are uniform with respect to $\epsilon$. Finally, using these energy estimates, we prove that the 
family of solutions $\left(w_{\epsilon}\right)_{\epsilon}$ of these regularized equations admits a limit $w$, which is a solution of Equation (1.1). We also show that this limiting solution $w$ has the same rate of decay as the regularized solution. We obtain the following theorem which describe the large time asymptotic behavior of the solutions of Equation (1.6) (the vorticity equation written in the scaled variables).

THEOREM 1.1. Let $T>0$ be a fixed time. There exist two positive constants $\bar{\alpha}_{0}$ and $\gamma_{0}$ such that, for all $\bar{\alpha} \leq \bar{\alpha}_{0}$, for all $W_{0}$ in $H^{2}(2)$ satisfying

$$
\left\|W_{0}\right\|_{H^{1}}^{2}+\bar{\alpha} e^{-\tau_{0}}\left\|\Delta W_{0}\right\|_{L^{2}}^{2}+\left\||\xi|^{2} W_{0}\right\|_{L^{2}}^{2}+\bar{\alpha} e^{-\tau_{0}}\left\||\xi|^{2} \Delta W_{0}\right\|_{L^{2}}^{2} \leq \gamma_{0},
$$

where $\tau_{0}=\log T$, Equation (1.6) has a unique solution $W(\tau) \in$ $C^{0}\left(\left[\tau_{0},+\infty\left[, H^{2}(2)\right)\right.\right.$ satisfying $W\left(\tau_{0}\right)=W_{0}$.

Moreover, the following inequality is satisfied, for all $\tau \geq \tau_{0}$,

$$
\left\|\left(1-\bar{\alpha} e^{-\tau} \Delta\right)(W(\tau)-\beta G)\right\|_{L^{2}(2)} \leq C e^{-\frac{\theta}{2} \tau}
$$

where $C$ and $\theta$ are positive constants, $\theta<\frac{1}{2}$, ,

$$
\beta=\int_{\mathbb{R}^{2}} W_{0}(\xi) d \xi
$$

and where $G$ is the Oseen vortex defined by

$$
G(\xi)=\frac{1}{4 \pi} e^{-|\xi|^{2} / 4}, \xi \in \mathbb{R}^{2} .
$$

The interpretation of the result in the unscaled variables $(x, t)$ is as follows: Let

$$
\Omega(x, t)=\frac{1}{t+T} G\left(\frac{x}{\sqrt{\nu(t+T)}}\right)
$$

and

$$
u^{\Omega}(x, t)=\sqrt{\frac{\nu}{t+T}} V^{G}\left(\frac{x}{\sqrt{\nu(t+T)}}\right) .
$$

From Theorem 1.1, we deduce the following result.

Corollary 1.2. Let $T>0$ be a fixed time so that $\frac{\alpha}{\nu T}<\bar{\alpha}_{0}$. Then, there exists a positive constant $\gamma$ such that for all $w_{0}$ in $H^{2}(2)$ satisfying

$$
T\left\|w_{0}\right\|_{L^{2}}^{2}+T^{\frac{3}{2}}\left\|\nabla w_{0}\right\|_{L^{2}}^{2}+\alpha T\left\|\Delta w_{0}\right\|_{L^{2}}^{2}+\left\|x^{2} w_{0}\right\|_{L^{2}}^{2}+\alpha\left\|x^{2} \Delta w_{0}\right\|_{L^{2}}^{2} \leq \gamma,
$$

the unique solution $w(x, t)$ of Equation (1.1) satisfies, for all $t \geq 0$,

$$
\begin{aligned}
& \left\|\left(1-\frac{\alpha}{T} \Delta\right)(w(t)-\beta \Omega)\right\|_{L^{p}} \leq C_{p}(T+t)^{-1-\frac{\theta}{2}+\frac{1}{p}}, 1 \leq p \leq 2, \\
& \left\|x^{2}\left(1-\frac{\alpha}{T} \Delta\right)(w(t)-\beta \Omega)\right\|_{L^{2}} \leq C(T+t)^{-1-\frac{\theta}{2}}
\end{aligned}
$$

where $C, C_{p}$ and $\theta, \theta<1 / 2$, are three positive constants and $\Omega$ is given by $(1.10)$. If $u(x, t)$ is the velocity field obtained from $w(x, t)$ via the Biot-Savart law, then

$$
\left\|\left(1-\frac{\alpha}{T} \Delta\right)\left(u(t)-\beta u^{\Omega}\right)\right\|_{L^{q}} \leq C_{q}(T+t)^{-\frac{1}{2}-\frac{\theta}{2}+\frac{1}{q}}, 1<q<\infty
$$

where $0<\theta<1 / 2, C_{q}>0$ is a constant and $u^{\Omega}$ is given by (1.11). 
We emphasize that, although the system $(S G F)$ converges to the system of the Navier-Stokes equations when $\alpha$ tends to zero, the results that we obtained here, are true for all values of $\alpha$ and not only for small values of $\alpha$.

Indeed, if we look at the rescaled equation (1.6), we remark that the coefficient $\bar{\alpha}$ can be as small as we want provided that we choose the parameter $T$ large enough. This shows that the Oseen vortex is an asymptotic solution of the system $(S G F)$.

We also note that the techniques that we use to prove Theorem 1.1 are quite different from the one used in the case of the Navier-Stokes equations ([16]). Actually, we use the method of energy functionals developed in [13] and [14]. As in [13] and [14], we introduce "primitives" of the function $W$. More precisely, we introduce the auxiliary function $F(\xi, \tau)$ given by

$$
F(\xi, \tau)=(-\Delta)^{-\frac{3}{4}}(W(\xi, \tau)-\beta G(\xi)) .
$$

This function $F(\xi, \tau)$ has a better decay rate than $W(\xi, \tau)-\beta G(\xi)$.

This paper is organized as follows. In the next section, we state the Biot-Savart law and recall some useful estimates of the velocity in terms of the vorticity. We also prove the local existence of the solution of the regularized vorticity equation in the space $H^{2}(m), m \geq 0$. In Section 3, we study some spectral properties of the operator $\mathcal{L}$, we decompose the solution $w$ and we give some auxiliary lemmas on the auxiliary function $F$ defined by (1.14). In section 4 , we introduce several functionals and we derive energy estimates in the space $H^{2}(2)$. We also state the theorem (see Theorem 3.3) which describes the first order asymptotics of small solutions of the regularized equation. The last section is devoted to the proof of Theorem 3.3. Finally, we pass to the limit when $\epsilon$ tends to zero and we prove Theorem 1.1 and Corollary 1.2 .

\section{Acknowledgements :}

I would like to express all my gratitude to Geneviève Raugel, without whom this work could not have been done.

\section{Preliminaries and regularized vorticity equation}

2.1. The Biot-Savart law. As we explained in the introduction, our approach consists first in studying the behavior of the solutions of the vorticity equation (1.1) and then, to derive information about the solutions of the system $(S G F)$. For this reason, we begin our study by recalling the relationship between the velocity field $u$ and the associated vorticity $w$. In two dimensions, the velocity field $u$ is defined in terms of the vorticity via the Biot-Savart law

$$
u(x)=\frac{1}{2 \pi} \int_{\mathbb{R}^{2}} \frac{(x-y)^{\perp}}{|x-y|^{2}} w_{3}(y) d y
$$

where $x^{\perp}=\left(-x_{2}, x_{1}\right)^{T}$ and $w=\left(0,0, w_{3}\right)$.

The following lemma collects useful estimates for the velocity $u$ in terms of the vorticity $w($ see $[\mathbf{1 6}])$.

LEMMA 2.1. Let $u$ be the velocity field obtained from $w$ via the Biot-Savart law (2.1). 
(a) Assume that $1<p<2<q<\infty$ and $\frac{1}{q}=\frac{1}{p}-\frac{1}{2}$. If $w \in L^{p}\left(\mathbb{R}^{2}\right)$, then $u \in L^{q}\left(\mathbb{R}^{2}\right)^{2}$, and there exists a positive constant $C_{p}$ such that

$$
\|u\|_{L^{q}} \leq C_{p}\|w\|_{L^{p}} .
$$

(b) Assume that $1 \leq p<2<q \leq \infty$ and define $\lambda \in(0,1)$ by the relation $\frac{1}{2}=$ $\frac{\lambda}{p}+\frac{1-\lambda}{q}$.

If $w \in L^{p}\left(\mathbb{R}^{2}\right) \cap L^{q}\left(\mathbb{R}^{2}\right)$, then $u \in L^{\infty}\left(\mathbb{R}^{2}\right)^{2}$, and there exists $C>0$ such that

$$
\|u\|_{L^{\infty}} \leq C\|w\|_{L^{p}}^{\lambda} .\|w\|_{L^{q}}^{1-\lambda} .
$$

(c) Assume that $1<p<\infty$. If $w \in L^{p}\left(\mathbb{R}^{2}\right)$, then $\nabla u \in L^{p}\left(\mathbb{R}^{2}\right)^{4}$, and there exists a positive constant $\tilde{C}_{p}$ such that

$$
\|\nabla u\|_{L^{p}} \leq \tilde{C}_{p}\|w\|_{L^{p}} .
$$

(d) Let $s \in \mathbb{R}$ and $J=(-\Delta)^{\frac{1}{2}}$.

If $J^{s-1} w \in L^{2}\left(\mathbb{R}^{2}\right)$, then $J^{s} u \in L^{2}\left(\mathbb{R}^{2}\right)^{2}$, and there exists a positive constant $C$ such that

$$
\left\|J^{s} u\right\|_{L^{2}} \leq C\left\|J^{s-1} w\right\|_{L^{2}} .
$$

In addition, div $u=0$ and $\operatorname{rot} u=\partial_{1} u_{2}-\partial_{2} u_{1}=w$.

For the proof of (a), (b) and (c), we refer to [16]. To prove (d), it is sufficient to write the expression of the Biot-Savart law in Fourier variables:

$$
\hat{u}(\eta)=\frac{i \eta^{\perp}}{|\eta|^{2}} \hat{w}(\eta) .
$$

LEMma 2.2. Let $u$ be the velocity field obtained from $w$ via the Biot-Savart law. There exists a positive constant $C$ such that, for any $w$ in $L^{2}(2) \cap H^{1}\left(\mathbb{R}^{2}\right)$, we have,

$$
\begin{aligned}
\text { (a) }\|u\|_{L^{\infty}} & \leq C\|w\|_{H^{1}}^{\frac{1}{2}}\|w\|_{L^{2}(2)}^{\frac{1}{2}}, \\
\text { (b) }\|u\|_{L^{4}} & \leq C\|w\|_{L^{2}(2)},
\end{aligned}
$$

Proof : In order to prove Inequality (a), we use Lemma 2.1, part (b), with $\lambda=\frac{1}{2}, p=\frac{6}{5}, q=6$, for example, and the fact that $H^{1}\left(\mathbb{R}^{2}\right)$ and $L^{2}(2)$ are continuously embedded into $L^{6}\left(\mathbb{R}^{2}\right)$ and $L^{\frac{6}{5}}\left(\mathbb{R}^{2}\right)$ respectively.

To prove Inequality (b), we use Lemma 2.1, part (a) and the continuous injection of $L^{2}(2)$ into $L^{\frac{4}{3}}\left(\mathbb{R}^{2}\right)$.

We remark that, according to the Biot-Savart law, the velocity is, in general, not in $L^{2}\left(\mathbb{R}^{2}\right)$. However, if $\int_{\mathbb{R}^{2}} w(\xi) d \xi=0$, then $u \in L^{2}\left(\mathbb{R}^{2}\right)$ and we have the following lemma.

Lemma 2.3. For any $w$ in $L^{2}(1)$, with $\int_{\mathbb{R}^{2}} w(\xi) d \xi=0$, the corresponding velocity field $u$ obtained from $w$ via the Biot-Savart law belongs to $L^{2}\left(\mathbb{R}^{2}\right)$ and we have:

$$
\|u\|_{L^{2}} \leq C\||\xi| w\|_{L^{2}} .
$$

where $C$ is a constant independent of $w$ and $u$. 
Proof : Let $\hat{w}$ be the Fourier transform of $w$. Then, using the Biot-Savart law and the fact that $\hat{w}(0)=0$, we can write

$$
\begin{aligned}
\|u\|_{L^{2}}^{2} & =\int_{\mathbb{R}^{2}} \frac{1}{|k|^{2}}|\hat{w}(k)|^{2} d k=\int_{0}^{1} \int_{\mathbb{R}^{2}}|\nabla \hat{w}(s k)|^{2} d k d s \\
& \leq C\|\nabla \hat{w}\|_{L^{2}}^{2} \leq C\||\xi| w\|_{L^{2}} .
\end{aligned}
$$

LEMmA 2.4. Let $h$ belongs to $L^{2}(1)$, then $(-\Delta)^{-\frac{1}{4}} h$ belongs to $L^{2}\left(\mathbb{R}^{2}\right)$ and we have

$$
\left\|(-\Delta)^{-\frac{1}{4}} h\right\|_{L^{2}} \leq C\|h\|_{L^{2}(1)}
$$

Proof : Using the Fourier transformation, we can write

$$
\begin{aligned}
\left\|(-\Delta)^{-\frac{1}{4}} h\right\|_{L^{2}}^{2} & =\int_{\mathbb{R}^{2}} \frac{1}{|k|}|\hat{h}(k)|^{2} d k \\
& \leq \int_{|k| \leq 1} \frac{1}{|k|}|\hat{h}(k)|^{2} d k+\int_{|k| \geq 1}|\hat{h}(k)|^{2} d k
\end{aligned}
$$

On one hand, the second term in this inequality can be bounded $\|h\|_{L^{2}}^{2}$. On the other hand, applying Hölder's inequality to the first term in the right-hand side of this inequality together with a classical Sobolev embedding theorem, we obtain

$$
\begin{aligned}
\left\|(-\Delta)^{-\frac{1}{4}} h\right\|_{L^{2}}^{2} & \leq\|\hat{h}\|_{L^{6}}^{2}\left(\int_{|k| \leq 1} \frac{1}{|k|^{\frac{3}{2}}} d k\right)^{\frac{2}{3}}+\|h\|_{L^{2}}^{2} \\
& \leq C\|\hat{h}\|_{H^{1}}^{2}+\|h\|_{L^{2}}^{2} \\
& \leq C\|(1+|\xi|) h\|_{L^{2}}^{2} \leq C\|h\|_{L^{2}(1)}^{2}
\end{aligned}
$$

REMARK 2.5. Using the Fourier transform, it is easy to remark that

$$
\left\|(-\Delta)^{-\frac{3}{4}} \partial_{i} h\right\|_{L^{2}} \leq\left\|(-\Delta)^{-\frac{1}{4}} h\right\|_{L^{2}}, i=1,2 .
$$

In fact, we have,

$$
\begin{aligned}
\left\|(-\Delta)^{-\frac{3}{4}} \partial_{i} h\right\|_{L^{2}}^{2} & =\int_{\mathbb{R}^{2}} \frac{\left|k_{i}\right|^{2}}{|k|^{3}}|\hat{h}(k)|^{2} d k \leq \int_{\mathbb{R}^{2}} \frac{1}{|k|}|\hat{h}(k)|^{2} d k \\
& =\left\|(-\Delta)^{-\frac{1}{4}} h\right\|_{L^{2}}^{2} .
\end{aligned}
$$

2.2. Local existence of the regularized vorticity equation. As we already said in the introduction, in order to study the solutions of Equation (1.1), in the weighted Sobolev spaces, we introduce the regularized equation (2.2) below. Indeed, since Equation (1.1) contains a nonlinearity that involves derivatives of order three, we cannot directly use classical methods of proofs to obtain the local existence. In order to overcome this difficulty, we add the smoothing term $\epsilon \Delta^{2}$. to 
the equation (1.1) and study the local well-posedness of the following equation in the space $H^{2}(m), m \geq 0$,

$$
\begin{aligned}
\partial_{t}\left(w_{\epsilon}-\alpha \Delta w_{\epsilon}\right)+\epsilon \Delta^{2} w_{\epsilon}-\nu \Delta w_{\epsilon}+u_{\epsilon} \cdot \nabla\left(w_{\epsilon}-\alpha \Delta w_{\epsilon}\right) & =0 \\
w_{\epsilon}(0) & =w_{0}
\end{aligned}
$$

THEOREM 2.6. Let $\epsilon>0$. There exists a time $t_{\max }>0$ such that, for all $w_{0} \in H^{2}(m), m \geq 0$, Equation (2.2) has a unique solution $w_{\epsilon}$ in the space $C^{0}\left(\left[0, t_{\max }\right], H^{2}(m)\right) \cap C^{1}\left(\left(0, t_{\max }\right], L^{2}(m)\right)$, with $w_{\epsilon}(0)=w_{0}$.

Proof : Let $\beta$ be a small positive constant (whose choice will be made more precise later). In order to prove the local existence of the solutions of Equation (2.2), we introduce the auxiliary variable and auxiliary unknown

$$
X=\beta x, \text { and } w_{\epsilon}(x, t)=s_{\epsilon}(\beta x, t)=s_{\epsilon}(X, t) .
$$

The equation satisfied by $s_{\epsilon}$ is given by

$$
\partial_{t}\left(s_{\epsilon}-\alpha \beta^{2} \Delta_{X} s_{\epsilon}\right)-\nu \beta^{2} \Delta_{X} s_{\epsilon}+\epsilon \beta^{4} \Delta_{X}^{2} s_{\epsilon}+\beta u_{\epsilon} \cdot \nabla_{X}\left(s_{\epsilon}-\alpha \beta^{2} \Delta_{X} s_{\epsilon}\right)=0
$$

We will see, in the proof of the local existence of solutions, that the above change of variables allows us to avoid restrictions on the size of $\alpha$.

Let $q(X)=\left(1+|X|^{2}\right)^{m / 2}$, and $z_{\epsilon}(t, X)=q(X) s_{\epsilon}(X, t)$.

Then $s_{\epsilon} \in C^{0}\left(\left[0, t_{\max }\right], H^{2}(m)\right) \cap C^{1}\left(\left(0, t_{\max }\right], L^{2}(m)\right)$ is a solution of Equation (2.3) if and only if the function $z_{\epsilon} \in C^{0}\left(\left[0, t_{\max }\right], H^{2}\left(\mathbb{R}^{2}\right)\right) \cap C^{1}\left(\left[0, t_{\max }\right], L^{2}\left(\mathbb{R}^{2}\right)\right)$ is a solution of the following equations

$$
\begin{aligned}
& \partial_{t}\left[z_{\epsilon}-\alpha \beta^{2} \Delta_{X} z_{\epsilon}-\alpha \beta^{2} q \Delta_{X}\left(q^{-1}\right) z_{\epsilon}-2 \alpha \beta^{2} q \nabla_{X}\left(q^{-1}\right) \nabla_{X} z_{\epsilon}\right]+\epsilon \beta^{4} \Delta_{X}^{2} z_{\epsilon} \\
& =P\left(z_{\epsilon}\right), \\
& z_{\epsilon}(0)=q(X) w_{0}(X)=z_{0} \in H^{2}\left(\mathbb{R}^{2}\right),
\end{aligned}
$$

where $P\left(z_{\epsilon}\right)=\nu \beta^{2} \Delta_{X} z_{\epsilon}+q(X)\left[\nu \beta^{2} \Delta_{X}\left(q^{-1}\right) z_{\epsilon}+2 \nu \beta^{2} \nabla_{X}\left(q^{-1}\right) \cdot \nabla_{X} z_{\epsilon}\right.$

$$
\begin{aligned}
& -\beta\left(u_{\epsilon} \cdot \nabla_{X}\right)\left(q^{-1} z_{\epsilon}\right)+\alpha \beta^{3}\left(u_{\epsilon} \cdot \nabla_{X}\right)\left(\Delta_{X}\left(q^{-1}\right) z_{\epsilon}+2 \nabla_{X}\left(q^{-1}\right) \cdot \nabla_{X}\left(z_{\epsilon}\right)\right) \\
& +\alpha \beta^{3}\left(u_{\epsilon} \cdot \nabla_{X}\right)\left(q^{-1} \Delta_{X} z_{\epsilon}\right)-\epsilon \beta^{4} \Delta_{X}\left(\Delta_{X}\left(q^{-1}\right) z_{\epsilon}+2 \nabla_{X}\left(q^{-1}\right) \cdot \nabla_{X} z_{\epsilon}\right) \\
& \left.-\epsilon \beta^{4}\left(\Delta_{X}\left(q^{-1}\right) \Delta_{X} z_{\epsilon}+2 \nabla_{X}\left(q^{-1}\right) \cdot \nabla_{X} \Delta_{X} z_{\epsilon}\right)\right] .
\end{aligned}
$$

Let $A$ be the linear operator: $D(A)=H^{2}\left(\mathbb{R}^{2}\right) \rightarrow L^{2}\left(\mathbb{R}^{2}\right)$, given by

$$
A=\alpha \beta^{2} \Delta_{X}+\alpha \beta^{2} q \Delta_{X}\left(q^{-1}\right)
$$

If we suppose that $\beta$ is small enough, then $I d-A$ is invertible from $L^{2}\left(\mathbb{R}^{2}\right)$ into $H^{2}\left(\mathbb{R}^{2}\right)$. Indeed, for $f \in L^{2}\left(\mathbb{R}^{2}\right)$, we consider the following problem.

Find $u \in H^{2}\left(\mathbb{R}^{2}\right)$ such that

$$
(I d-A) u=f
$$

Let $a$ be the bilinear form defined, for $u, v \in H^{1}\left(\mathbb{R}^{2}\right)$, by

$$
\begin{aligned}
a(u, v) & =\int_{\mathbb{R}^{2}} u(X) \cdot v(X) d X+\alpha \beta^{2} \int_{\mathbb{R}^{2}} \nabla u(X) \cdot \nabla v(X) d X \\
& -\alpha \beta^{2} \int_{\mathbb{R}^{2}} q \Delta\left(q^{-1}\right) u(X) v(X) d X
\end{aligned}
$$


Then, we have, for any $u \in H^{1}\left(\mathbb{R}^{2}\right)$,

$$
\begin{gathered}
a(u, u) \geq \int_{\mathbb{R}^{2}}|u(X)|^{2} d X+\alpha \beta^{2} \int_{\mathbb{R}^{2}}|\nabla u(X)|^{2} d X \\
-\alpha \beta^{2} \int_{\mathbb{R}^{2}}\left|q \Delta\left(q^{-1}\right) \| u(X)\right|^{2} d x
\end{gathered}
$$

We notice that $\left|q \Delta\left(q^{-1}\right)\right| \leq C_{0}$, where $C_{0}>0$ is a constant.

Thus,

$$
a(u, u) \geq\left(1-\alpha \beta^{2} C_{0}\right)\|u\|_{L^{2}}^{2}+\alpha \beta^{2}\|\nabla u\|_{L^{2}}^{2} .
$$

Supposing that $\beta$ is small enough such that $1-\alpha \beta^{2} C_{0}>0$, we obtain from the LaxMilgram theorem that there exists a unique solution $u \in H^{1}\left(\mathbb{R}^{2}\right)$ of $(2.5)$. Since $f$ belongs to $L^{2}\left(\mathbb{R}^{2}\right)$, we deduce from a classical regularity theorem that $u$ belongs to $H^{2}\left(\mathbb{R}^{2}\right)$ and

$$
\|u\|_{H^{2}} \leq C_{\beta}\|f\|_{L^{2}},
$$

where $C_{\beta}>0$ is a constant, independent of $f$ and $u$.

On the other hand, if $\beta$ is small enough, we can show by the same way that $\left(I d-A-\alpha \beta^{2} q \nabla\left(q^{-1}\right) \nabla\right)^{-1}$ exists and is defined from $L^{2}\left(\mathbb{R}^{2}\right)$ into $H^{2}\left(\mathbb{R}^{2}\right)$.

In fact, remarking that $\left|q \nabla\left(q^{-1}\right)\right| \leq C_{1}$, where $C_{1}>0$, we have

$$
\begin{aligned}
& a(u, u)-\alpha \beta^{2} \int_{\mathbb{R}^{2}} q \nabla\left(q^{-1}\right) \nabla u(X) u(X) d X \geq \\
& \quad\left(1-\alpha \beta^{2} C_{0}\right)\|u\|_{L^{2}}^{2}+\alpha \beta^{2}\|\nabla u\|_{L^{2}}^{2}-\alpha \beta^{2} C_{1}\|\nabla u\|_{L^{2}}\|u\|_{L^{2}}
\end{aligned}
$$

Then, applying the Young inequality and supposing that $1-\alpha \beta^{2} C_{0}-\frac{1}{2} \alpha \beta^{2} C_{1}^{2}>0$, we obtain,

$$
\begin{aligned}
& a(u, u)-\alpha \beta^{2} \int_{\mathbb{R}^{2}} q \nabla\left(q^{-1}\right) \nabla u(x) u(x) d x \geq \\
& \quad\left(1-\alpha \beta^{2} C_{0}-\frac{1}{2} \alpha \beta^{2} C_{1}^{2}\right)\|u\|_{L^{2}}^{2}+\frac{1}{2} \alpha \beta^{2}\|\nabla u\|_{L^{2}}^{2}
\end{aligned}
$$

Finally, using the Lax-Milgram theorem, we can show that the operator $\left(I d-A-\alpha \beta^{2} q \nabla\left(q^{-1}\right) \nabla\right)$ is invertible.

Now, let $B=-\alpha \beta^{2} q \nabla\left(q^{-1}\right) \nabla$.

Then, Equation (2.4) can be written as

$$
\partial_{t} z_{\epsilon}+\epsilon(I-A+B)^{-1} \Delta_{X}^{2} z_{\epsilon}=(I-A+B)^{-1} P\left(z_{\epsilon}\right) \equiv \tilde{P}\left(z_{\epsilon}\right)
$$

The operator $(I-A+B)^{-1} \Delta_{X}^{2}$ can be defined from $H^{2}\left(\mathbb{R}^{2}\right)$ into $L^{2}\left(\mathbb{R}^{2}\right)$ and can be written as

$$
(I-A+B)^{-1} \Delta_{X}^{2}=(I-A)^{-1} \Delta_{X}^{2}-(I-A+B)^{-1} B(I-A)^{-1} \Delta_{X}^{2}
$$

The operator $(I-A)^{-1} \Delta_{X}^{2}$ defined from $H^{2}\left(\mathbb{R}^{2}\right)$ into $L^{2}\left(\mathbb{R}^{2}\right)$ is self-adjoint and positive, thus, $-(I-A)^{-1} \Delta_{X}^{2}$ is the generator of an analytic semigroup in $L^{2}\left(\mathbb{R}^{2}\right)$. Let

$$
R=-(I-A+B)^{-1} B(I-A)^{-1} \Delta_{X}^{2}: H^{1}\left(\mathbb{R}^{2}\right) \longrightarrow L^{2}\left(\mathbb{R}^{2}\right) .
$$

Then, there exists a constant $C_{\beta}>0$ such that, for all $u \in H^{1}\left(\mathbb{R}^{2}\right)$,

$$
\|R u\|_{L^{2}} \leq C_{\beta}\|u\|_{H^{1}} \text {. }
$$

Therefore, $(I-A)^{-1} \Delta_{X}^{2}+R$ is the generator of an analytic semigroup in $L^{2}\left(\mathbb{R}^{2}\right)$ (see [22] Corollary 2.2 page 81 ).

Since $\tilde{P}\left(z_{\epsilon}\right)$ is a locally Lipschitz continuous mapping from $H^{1}\left(\mathbb{R}^{2}\right)$ into $L^{2}\left(\mathbb{R}^{2}\right)$, we deduce, from a classical result $([\mathbf{2 2}],[\mathbf{4}])$ that there exists a time $t_{\max }>0$ such that, for all $z_{0} \in H^{2}\left(\mathbb{R}^{2}\right)$, Equation (2.4) has a unique classical solution 
$z_{\epsilon} \in C^{0}\left(\left[0, t_{\max }\right], H^{2}\left(\mathbb{R}^{2}\right)\right) \cap C^{1}\left(\left(0, t_{\max }\right], L^{2}\left(\mathbb{R}^{2}\right)\right)$ satisfying $z_{\epsilon}(0)=z_{0}$. Therefore, we have proved the local existence and uniqueness of solutions of Equation (2.3) in the space $C^{0}\left(\left[0, t_{\max }\right], H^{2}(m)\right) \cap C^{1}\left(\left(0, t_{\max }\right], L^{2}(m)\right)$ and thus, of the regularized vorticity equation $(2.2)$.

To complete the proof of the local existence of the solution $w$ of Equation (1.1), we need to take the limit of $w_{\epsilon}$ when $\epsilon$ tends to zero, but we do not know how to prove that the existence time of $w_{\epsilon}$ is independent of $\epsilon$. Therefore in the next sections, the study of the asymptotic behavior will be done for $w_{\epsilon}$. We will establish energy estimates on $w_{\epsilon}$, which are uniform in $\epsilon$. Then passing to the limit when $\epsilon$ tends to zero, we will prove that the limit of $w_{\epsilon}$ is the solution of Equation (1.1) and satisfies the same energy estimates as $w_{\epsilon}$.

\section{Spectral study of the operator $\mathcal{L}$ and decomposition of the solution}

One of the main ideas in our analysis of the long-time asymptotics of Equation (2.2) is based on rewriting this equation in terms of the scaled variables $(\xi, \tau)$ given by (1.2). We recall that the new functions $W_{\epsilon}$ and $V_{\epsilon}$ in the rescaled variables are given by

$$
w_{\epsilon}(x, t)=\frac{1}{t+T} W_{\epsilon}\left(\frac{x}{\sqrt{\nu(t+T)}}, \log (t+T)\right)
$$

and

$$
u_{\epsilon}(x, t)=\sqrt{\frac{\nu}{t+T}} V_{\epsilon}\left(\frac{x}{\sqrt{\nu(t+T)}}, \log (t+T)\right)
$$

where $w_{\epsilon}(x, t)$ is a solution of $(2.2)$ and $u_{\epsilon}(x, t)$ is the corresponding velocity field. We remark that $t=0$ corresponds to $\tau_{0}=\log T$.

The "rescaled vorticity" $W_{\epsilon}(\xi, \tau)$ satisfies the following system

$$
\begin{aligned}
\partial_{\tau}\left(W_{\epsilon}-\bar{\alpha} e^{-\tau} \Delta_{\xi} W_{\epsilon}\right)- & \mathcal{L} W_{\epsilon}+V_{\epsilon} \cdot \nabla_{\xi}\left(W_{\epsilon}-\bar{\alpha} e^{-\tau} \Delta_{\xi} W_{\epsilon}\right)+\bar{\epsilon} e^{-2 \tau} \Delta_{\xi}^{2} W_{\epsilon} \\
& +\bar{\alpha} e^{-\tau} \Delta_{\xi} W_{\epsilon}+\frac{\bar{\alpha}}{2} e^{-\tau} \xi \cdot \nabla_{\xi} \Delta_{\xi} W_{\epsilon}=0, \\
W_{\epsilon}\left(\xi, \tau_{0}\right)=W_{0}(\xi)= & T w_{0}(x),
\end{aligned}
$$

where the operator $\mathcal{L}$ has been defined in (1.7), $\tau_{0}, \bar{\alpha}, \bar{\epsilon}$ are given by (1.5) and, according to the Biot-Savart law,

$$
V_{\epsilon}(\xi, \tau)=\frac{1}{2 \pi} \int_{\mathbb{R}^{2}} \frac{(\xi-\eta)^{\perp}}{|\xi-\eta|^{2}} W_{3}^{\epsilon}(\eta, \tau) d \eta
$$

where $W_{\epsilon}=\left(0,0, W_{3}^{\epsilon}\right)$.

We point out that Equation (3.3) preserves the total mass of $W_{\epsilon}$. Indeed, integrating (3.3) over $\mathbb{R}^{2}$, and using the fact that $\operatorname{div} V_{\epsilon}=0$, we obtain

$$
\int_{\mathbb{R}^{2}} W_{\epsilon}(\xi, \tau) d \xi=\int_{\mathbb{R}^{2}} W_{0}(\xi) d \xi .
$$


3.1. The operator $\mathcal{L}$. As we already explained in the introduction, working in weighted Sobolev spaces allows to push the continuous spectrum of $\mathcal{L}$ to the left. If one studies the spectrum of the operator $\mathcal{L}$ acting on $L^{2}(m)$, one finds that it consists of a sequence of eigenvalues

$$
\sigma_{d}=\left\{-\frac{k}{2} \mid k=0,1,2, \ldots, m-2\right\}
$$

and continuous spectrum

$$
\sigma_{c}=\left\{\lambda \in \mathbb{C} \mid \operatorname{Re}(\lambda) \leq-\frac{m-1}{2}\right\}
$$

(for more details, see Appendix A in [16]).

The eigenvectors corresponding to the isolated eigenvalues can be explicitly computed and are rapidly decaying at infinity (see Appendix B in [16]).

In our study, we will consider the behavior of small solutions of (3.3) in the space $L^{2}(m)$ with $m=2$. The operator $\mathcal{L}$ has a simple isolated eigenvalue $\lambda_{0}=0$ in $L^{2}(2)$, with corresponding eigenfunction

$$
G(\xi)=\frac{1}{4 \pi} e^{-|\xi|^{2} / 4}, \xi \in \mathbb{R}^{2} .
$$

For any $\beta \in \mathbb{R}$,

$$
W(\xi)=\beta G(\xi)
$$

is called the Oseen vortex. The corresponding velocity field $V^{G}$ (such that $\operatorname{rot} V^{G}=$ $G)$ is given by

$$
V^{G}(\xi)=\frac{1-e^{-|\xi|^{2} / 4}}{2 \pi|\xi|^{2}}\left(\begin{array}{r}
-\xi_{2} \\
\xi_{1}
\end{array}\right)
$$

REMARK 3.1. It is clear that $\xi$ is orthogonal to $V^{G}$, therefore

$$
V^{G} \cdot \nabla G(\xi)=0
$$

and

$$
V^{G} \cdot \nabla \Delta G(\xi)=0
$$

As a consequence of the equality (3.8), the Oseen vortex defined by (3.6) is a stationary solution of the equation

$$
\partial_{\tau} W-\mathcal{L} W+V . \nabla W=0
$$

where $V$ is the velocity field corresponding to the vorticity $W$.

Note that this equation is precisely the vorticity equation corresponding to the Navier-Stokes equations in space dimension two.

REMARK 3.2. Remarking that $\left|V^{G}\right| \sim|\xi|^{-1}$ as $|\xi| \rightarrow+\infty$, we obtain that $V^{G} \in L^{q}\left(\mathbb{R}^{2}\right)^{2}, \forall q>2$. 
3.2. Decomposition of the solution and auxiliary lemmas. In this paper, we will prove the following result.

THEOREM 3.3. Let $T>0$ be a fixed time. There exist two positive constants $\bar{\alpha}_{0}$ and $\gamma_{0}$ such that for all $\bar{\alpha} \leq \bar{\alpha}_{0}$ and for all $W_{0}$ in $H^{2}(2)$ satisfying

$$
\left\|W_{0}\right\|_{H^{1}}^{2}+\bar{\alpha} e^{-\tau_{0}}\left\|\Delta W_{0}\right\|_{L^{2}}^{2}+\left\||\xi|^{2} W_{0}\right\|_{L^{2}}^{2}+\bar{\alpha} e^{-\tau_{0}}\left\||\xi|^{2} \Delta W_{0}\right\|_{L^{2}}^{2} \leq \gamma_{0},
$$

where $\tau_{0}=\log T$, Equation (3.3) has a unique solution $W_{\epsilon}(\tau) \in$ $C^{0}\left(\left[\tau_{0},+\infty\left[, H^{2}(2)\right)\right.\right.$ satisfying $W_{\epsilon}\left(\tau_{0}\right)=W_{0}$. Moreover, the following inequality is satisfied, for all $\tau \geq \tau_{0}$,

$$
\left\|\left(1-\bar{\alpha} e^{-\tau} \Delta\right)\left(W_{\epsilon}(\tau)-\beta G\right)\right\|_{L^{2}(2)}^{2} \leq C e^{-\theta \tau}
$$

where $C$ and $\theta, \theta<\frac{1}{2}$, are positive constants, $\beta=\int_{\mathbb{R}^{2}} W_{0}(\xi) d \xi$ and $G$ is the Oseen vortex defined by (3.6).

Since we are interested in studying the behavior of the solution of (3.3) with initial data $W_{0}$ near an Oseen vortex in the space $H^{2}(2)$, it is convenient to introduce the following change of functions:

$$
W_{\epsilon}(\xi, \tau)=\beta G(\xi)+f_{\epsilon}(\xi, \tau),
$$

where, due to the conservation of mass property (3.4),

$$
\beta \equiv \int_{\mathbb{R}^{2}} W_{0}(\xi) d \xi=\beta .
$$

Thus, $f_{\epsilon}(\xi, \tau)$ belongs to $L_{0}^{2}(2)$ for any $\tau \geq 0$, where $L_{0}^{2}(m)=\left\{f \in L^{2}(m) / \int_{\mathbb{R}^{2}} f(\xi) d \xi=0\right\}$.

In what follows, we will drop the index $\epsilon$ and simply denote $f_{\epsilon}(x, t)$ by $f(x, t)$.

Taking into account the properties (3.8) and (3.9), we see that $f(\xi, \tau)$ satisfies the following equation

$$
\begin{aligned}
& \partial_{\tau}\left(f-\bar{\alpha} e^{-\tau} \Delta f\right)-\mathcal{L} f+\bar{\epsilon} e^{-2 \tau} \Delta^{2} f+\bar{\alpha} e^{-\tau} \Delta f+\frac{\bar{\alpha}}{2} e^{-\tau} \xi . \nabla \Delta f \\
& +K_{f} \cdot \nabla\left(f-\bar{\alpha} e^{-\tau} \Delta f\right)+\beta V^{G} \cdot \nabla\left(f-\bar{\alpha} e^{-\tau} \Delta f\right)+\beta K_{f} \cdot \nabla\left(G-\bar{\alpha} e^{-\tau} \Delta G\right) \\
& +\bar{\epsilon} \beta e^{-2 \tau} \Delta^{2} G+\beta \bar{\alpha} e^{-\tau} \Delta G+\beta \frac{\bar{\alpha}}{2} e^{-\tau} \xi . \nabla \Delta G=0
\end{aligned}
$$

where $K_{f}(\xi, \tau)=V(\xi, \tau)-\beta V^{G}$.

As we explained in the introduction, the main argument in the study of the asymptotic behavior of $f$ is the use of functional method. This method consists in writing various energy estimates for $f$ and in considering a linear combination of these functionals in order to establish that $f$ converges to zero with an exponential decay rate in the space $H^{2}(2)$.

In what follows, we will establish various energy estimates for $f(\xi, \tau)$ in the spaces $H^{2}(2)$. So, in a first step, we will control the $L^{2}$-norm of $f$. Unfortunately, we cannot obtain good estimates of $f$ in the space $L^{2}\left(\mathbb{R}^{2}\right)$.

Indeed, if $(.,$.$) denotes the scalar product in L^{2}\left(\mathbb{R}^{2}\right)$, we have,

$$
(-\mathcal{L} f, f)=\|\nabla f\|_{L^{2}}^{2}-\frac{1}{2}\|f\|_{L^{2}}^{2}
$$


which is not helpful in the functional method.

In the paper [13] (where the asymptotic behavior was studied in the one-dimensional space), the authors considered the primitive of $f$, because it had a better decay than $f$. In our study, since we are in the two-dimensional case, we will introduce the function

$$
F(\xi, \tau)=(-\Delta)^{-\frac{3}{4}} f(\xi, \tau)
$$

Before continuing our analysis, we give the following lemmas that will be useful later.

Lemma 3.4. Let $f$ belong to $L^{2}(2)$ such that $\int_{\mathbb{R}^{2}} f(\xi) d \xi=0$, then $(-\Delta)^{-\frac{3}{4}} f$ belongs to $L^{2}\left(\mathbb{R}^{2}\right)$ and we have

$$
\left\|(-\Delta)^{-\frac{3}{4}} f\right\|_{L^{2}} \leq C\|f\|_{L^{2}(2)},
$$

where $C>0$ is independent of $f$.

Proof : Let $\hat{f}$ be the Fourier transform of $f$ given by

$$
\hat{f}(k)=\int_{\mathbb{R}^{2}} f(\xi) \exp (-i k \cdot \xi) d \xi .
$$

Since $\hat{f}(0)=0$, we can write

$$
\begin{aligned}
\left\|(-\Delta)^{-\frac{3}{4}} f\right\|_{L^{2}}^{2} & =\int_{\mathbb{R}^{2}} \frac{1}{|k|^{3}}|\hat{f}(k)|^{2} d k \\
& \leq \int_{|k| \leq 1} \frac{1}{|k|^{3}}|\hat{f}(k)|^{2} d k+\int_{|k| \geq 1}|\hat{f}(k)|^{2} d k \\
& \leq \int_{0}^{1} \int_{|k| \leq 1} \frac{1}{|k|}|\nabla \hat{f}(s k)|^{2} d k d s+\|f\|_{L^{2}}^{2}
\end{aligned}
$$

Applying Hölder's inequality on the first term in the above inequality, we obtain

$$
\begin{gathered}
\left\|(-\Delta)^{-\frac{3}{4}} f\right\|_{L^{2}}^{2} \leq \int_{0}^{1}\left(\int_{|k| \leq 1}|\nabla \hat{f}(s k)|^{6} d k\right)^{\frac{1}{3}}\left(\int_{|k| \leq 1} \frac{1}{|k|^{\frac{3}{2}}} d k\right)^{\frac{2}{3}} d s+\|f\|_{L^{2}}^{2} \\
\leq C\|\nabla \hat{f}\|_{L^{6}}^{2}+\|f\|_{L^{2}}^{2} \leq C\|\nabla \hat{f}\|_{H^{1}}^{2}+\|f\|_{L^{2}}^{2}
\end{gathered}
$$

Therefore,

$$
\left\|(-\Delta)^{-\frac{3}{4}} f\right\|_{L^{2}}^{2} \leq C\left\|\left(1+|\xi|^{2}\right) f\right\|_{L^{2}}^{2} \leq C\|f\|_{L^{2}(2)}^{2}
$$

We emphasize that in order to bound the $L^{2}$-norm of $f$, it is sufficient to bound the $L^{2}$-norms of $\nabla F$ and of $\nabla f$.

In fact, applying Hölder's inequality, we have

$$
\|f\|_{L^{2}}^{2}=\int_{\mathbb{R}^{2}}(-\Delta)^{\frac{1}{4}} f(-\Delta)^{\frac{1}{2}}(-\Delta)^{\frac{-3}{4}} f \leq\left\|(-\Delta)^{\frac{1}{4}} f\right\|_{L^{2}}\left\|(-\Delta)^{\frac{1}{2}} F\right\|_{L^{2}}
$$


Then, using the fact that $\left\|(-\Delta)^{\frac{1}{2}} F\right\|_{L^{2}}=\|\nabla F\|_{L^{2}}$ and applying Young's inequality, we obtain

$$
\begin{aligned}
\|f\|_{L^{2}}^{2} & \leq\|f\|_{L^{2}}^{\frac{1}{2}}\left\|(-\Delta)^{\frac{1}{2}} f\right\|_{L^{2}}^{\frac{1}{2}}\|\nabla F\|_{L^{2}} \leq\|f\|_{L^{2}}^{\frac{1}{2}}\|\nabla f\|_{L^{2}}^{\frac{1}{2}}\|\nabla F\|_{L^{2}} \\
& \leq \frac{1}{2}\|\nabla F\|_{L^{2}}^{2}+\frac{1}{4}\|\nabla f\|_{L^{2}}^{2}+\frac{1}{4}\|f\|_{L^{2}}^{2}
\end{aligned}
$$

Thus,

$$
\|f\|_{L^{2}}^{2} \leq \frac{2}{3}\|\nabla F\|_{L^{2}}^{2}+\frac{1}{3}\|\nabla f\|_{L^{2}}^{2}
$$

In order to bound the $L^{2}$-norm of $\nabla F$, the first step consists in writing the equation satisfied by $F$. The lemma stated below will help us to write this equation.

Lemma 3.5. Let $F$ be given by (3.16). We have

$$
\left((-\Delta)^{-\frac{3}{4}}(\xi . \nabla f)\right)=-\frac{3}{2} F+\xi . \nabla F
$$

and

$$
(-\Delta)^{-\frac{3}{4}} \mathcal{L} f=\Delta F+\frac{1}{2} \xi . \nabla F+\frac{1}{4} F .
$$

Proof : In what follows, the Fourier transform of $f$ is sometimes denoted by $P(f)$. Using the Fourier transformation and integrating by parts, we can write

$$
\begin{gathered}
P\left((-\Delta)^{-\frac{3}{4}}(\xi \cdot \nabla f)\right)(k)=-2 P\left((-\Delta)^{-\frac{3}{4}} f\right)(k)+\frac{i k_{i}}{|k|^{\frac{3}{2}}} \int_{\mathbb{R}^{2}} \xi_{i} f(\xi) \exp (-i k \cdot \xi) d \xi \\
=-2 P\left((-\Delta)^{-\frac{3}{4}} f\right)(k)-\frac{k_{i}}{|k|^{\frac{3}{2}}} \partial_{k_{i}} \int_{\mathbb{R}^{2}} f(\xi) \exp (-i k \cdot \xi) d \xi \\
=-\frac{3}{2} P\left((-\Delta)^{-\frac{3}{4}} f\right)(k)+P\left(\xi \cdot \nabla(-\Delta)^{-\frac{3}{4}} f\right)(k)
\end{gathered}
$$

Thus,

$$
(-\Delta)^{-\frac{3}{4}} \mathcal{L} f=\Delta F+\frac{1}{2} \xi . \nabla F+\frac{1}{4} F .
$$

Lemma 3.5 at once implies that

$$
\left(-(-\Delta)^{-\frac{3}{4}} \mathcal{L} f, F\right)=\|\nabla F\|_{L^{2}}^{2}+\frac{1}{4}\|F\|_{L^{2}}^{2},
$$

which will help us in obtaining "good $L^{2}$-estimates" on $F$.

Using Lemma 3.5, we see that $F$ satisfies the following equation

$$
\begin{aligned}
& \partial_{\tau}\left(F-\bar{\alpha} e^{-\tau} \Delta F\right)-\Delta F-\frac{1}{2} \xi \cdot \nabla F-\frac{1}{4} F+\bar{\epsilon} e^{-2 \tau} \Delta^{2} F+\frac{\bar{\alpha}}{4} e^{-\tau} \Delta F \\
& +\frac{\bar{\alpha}}{2} e^{-\tau} \xi \cdot \nabla \Delta F+(-\Delta)^{-\frac{3}{4}}\left(\left(K_{f}+\beta V_{G}\right) \cdot \nabla\left((-\Delta)^{\frac{3}{4}} F+\bar{\alpha} e^{-\tau}(-\Delta)^{\frac{7}{4}} F\right)\right) \\
& +\beta(-\Delta)^{-\frac{3}{4}}\left(K_{f} \cdot \nabla\left(G-\bar{\alpha} e^{-\tau} \Delta G\right)\right. \\
& +\frac{\bar{\alpha}}{2} e^{-\tau} \xi \cdot \nabla(-\Delta)^{\frac{1}{4}} G+\bar{\epsilon} \beta e^{-2 \tau}(-\Delta)^{\frac{5}{4}} G+\frac{\bar{\alpha} \beta}{4} e^{-\tau}(-\Delta)^{\frac{1}{4}} G=0
\end{aligned}
$$




\section{Asymptotic behavior of solutions and energy estimates}

In this section, we will establish various energy estimates of the solutions of Equation (3.14) in the space $H^{2}(2)$.

In what follows, we introduce a positive constant $\delta_{0}$ (that will be fixed later) and let $0<\delta \leq \delta_{0}$. We also assume that, for some $\tau_{0}<\tau_{1}<\hat{\tau}$, where $\hat{\tau}=\log \left(T+t_{\max }\right)$, we are given a solution $\left.f \in C^{(}\left[\tau_{0}, \tau_{1}\right], H^{2}(2)\right)$ of Equation (3.14) satisfying the following bound

$$
|\beta|^{2}+\|f(\tau)\|_{H^{1}}^{2}+\left\|\xi^{2} f(\tau)\right\|_{L^{2}}^{2}<\delta, \tau_{0} \leq \tau \leq \tau_{1}
$$

where $\beta$ is given by (3.13).

Our aim is to control the behavior of the solution $f$ on the time interval $\left[\tau_{0}, \tau_{1}\right]$, using energy functionals.

4.1. Energy estimates in $L^{2}\left(\mathbb{R}^{2}\right)$. In this section, we will introduce several functionals in order to establish energy estimates of $f$ in various "unweighted" Sobolev spaces, in particular in the space $H^{2}\left(\mathbb{R}^{2}\right)$.

For this purpose, we will bound $\|f(\tau)\|_{H^{1}}^{2}+\bar{\alpha} e^{-\tau}\|\Delta f(\tau)\|_{L^{2}}^{2}$. As we have explained in the previous section, it is essential to bound the $L^{2}$-norm of $\nabla F$ in order to bound the $L^{2}$-norm of $f$.

4.1.1. Estimates of $F$ and $\nabla F$. Let $F$ be given by (3.16). In order to estimate $\|F\|_{L^{2}}$ and $\|\nabla F\|_{L^{2}}$, we introduce our first functional $E_{0}(\tau)$ given by

$$
E_{0}(\tau)=\frac{1}{2}\left(\|F(\tau)\|_{L^{2}}^{2}+\bar{\alpha} e^{-\tau}\|\nabla F(\tau)\|_{L^{2}}^{2}\right)
$$

We have the following lemma for the functional $E_{0}$.

Lemma 4.1. Assume that $f \in L^{\infty}\left(\left[\tau_{0}, \tau_{1}\right], H^{2}(2)\right)$ is a solution of (3.14) satisfying the bound (4.1). Then, there exists two positive constants $C_{0}$ and $\theta, \theta<\frac{1}{2}$, such that for all $\tau \in\left[\tau_{0}, \tau_{1}\right]$,

$$
\begin{aligned}
\partial_{\tau} E_{0}(\tau)+ & \theta E_{0}(\tau)+\left(1+\frac{3 \bar{\alpha}}{4} e^{-\tau}-\frac{\theta \bar{\alpha}}{2} e^{-\tau}\right)\|\nabla F\|_{L^{2}}^{2}+\bar{\epsilon}\left(\frac{3}{4}-\frac{\theta}{2}\right) e^{-2 \tau}\|\Delta F\|_{L^{2}}^{2} \\
\leq & C_{0}|\beta|^{2}\left(\bar{\alpha}^{2}+\bar{\epsilon}\right) e^{-2 \tau}+C_{0}|\beta|^{2}\|\nabla f\|_{L^{2}}^{2} \\
& +C_{0}\left(\delta+|\beta|^{2}\right)\left(\|f\|_{L^{2}}^{2}+\left\||\xi|^{2} f\right\|_{L^{2}}^{2}\right) \\
& +C_{0}\left(\delta+|\beta|^{2}\right) \bar{\alpha}^{2} e^{-2 \tau}\left(\|\Delta f\|_{L^{2}}^{2}+\left\||\xi|^{2} \Delta f\right\|_{L^{2}}^{2}\right)
\end{aligned}
$$

Proof : In order to estimate the $L^{2}$-norm of $F$, we take the scalar product in $L^{2}\left(\mathbb{R}^{2}\right)$ of Equation (3.20) with $\mathrm{F}$. We obtain

$$
\begin{aligned}
& \partial_{\tau} E_{0}(\tau)+\frac{1}{4}\|F\|_{L^{2}}^{2}+\left(1-\frac{\bar{\alpha}}{4} e^{-\tau}\right)\|\nabla F\|_{L^{2}}^{2}+\frac{\bar{\alpha}}{2} e^{-\tau}(\xi \cdot \nabla \Delta F, F) \\
& +\bar{\epsilon} e^{-2 \tau}\|\Delta F\|_{L^{2}}^{2}+\frac{\bar{\alpha} \beta}{2} e^{-\tau}\left(\xi \cdot \nabla(-\Delta)^{\frac{1}{4}} G, F\right)+\bar{\epsilon} \beta e^{-2 \tau}\left((-\Delta)^{\frac{5}{4}} G, F\right) \\
& +\frac{\bar{\alpha} \beta}{4} e^{-\tau}\left((-\Delta)^{\frac{1}{4}} G, F\right)+I_{1}+I_{2}+I_{3}=0
\end{aligned}
$$

where

$$
I_{1}=\left((-\Delta)^{-\frac{3}{4}}\left(K_{f} \cdot \nabla\left((-\Delta)^{\frac{3}{4}} F+\bar{\alpha} e^{-\tau}(-\Delta)^{\frac{7}{4}} F\right)\right), F\right),
$$




$$
\begin{aligned}
& I_{2}=\beta\left((-\Delta)^{-\frac{3}{4}}\left(K_{f} \cdot \nabla\left(G-\bar{\alpha} e^{-\tau} \Delta G\right)\right), F\right), \\
& I_{3}=\beta\left((-\Delta)^{-\frac{3}{4}}\left(\left(V^{G} \cdot \nabla\left((-\Delta)^{\frac{3}{4}} F+\bar{\alpha} e^{-\tau}(-\Delta)^{\frac{7}{4}} F\right)\right), F\right) .\right.
\end{aligned}
$$

First, integrating by parts several times, we remark that

$$
\begin{aligned}
& (\xi . \nabla \Delta F, F)=-2 \int_{\mathbb{R}^{2}} \Delta F F d \xi-\int_{\mathbb{R}^{2}} \xi . \nabla F \Delta F d \xi \\
& \quad=2\|\nabla F\|_{L^{2}}^{2}+\sum_{k=1}^{2} \int_{\mathbb{R}^{2}} \xi . \nabla \partial_{k} F \partial_{k} F d \xi+\sum_{k=1}^{2} \int_{\mathbb{R}^{2}}\left|\partial_{k} F\right|^{2} d \xi \\
& \quad=2\|\nabla F\|_{L^{2}}^{2}
\end{aligned}
$$

Next, integrating by parts, we obtain the following estimates:

$$
\begin{aligned}
\left(\xi . \nabla(-\Delta)^{\frac{1}{4}} G, F\right) & \leq\left\||\xi| \nabla(-\Delta)^{\frac{1}{4}} G\right\|_{L^{2}}\|F\|_{L^{2}} \leq C\|F\|_{L^{2}}, \\
\left((-\Delta)^{\frac{1}{4}} G, F\right) & \leq\left\|(-\Delta)^{\frac{1}{4}} G\right\|_{L^{2}}\|F\|_{L^{2}} \leq C\|F\|_{L^{2}}, \\
\left((-\Delta)^{\frac{5}{4}} G, F\right) & =-\left((-\Delta)^{\frac{1}{4}} G, \Delta F\right) \leq\left\|(-\Delta)^{\frac{1}{4}} G\right\|_{L^{2}}\|\Delta F\|_{L^{2}} \\
& \leq C\|\Delta F\|_{L^{2}} .
\end{aligned}
$$

It remains to bound the terms $I_{1}, I_{2}$ and $I_{3}$. Using the fact that $\operatorname{div} K_{f}=0$, we can write

$$
I_{1}=\left(\sum_{i=1}^{2}(-\Delta)^{-\frac{3}{4}} \partial_{i}\left(K_{f}^{i}\left((-\Delta)^{\frac{3}{4}} F+\bar{\alpha} e^{-\tau}(-\Delta)^{\frac{7}{4}} F\right)\right), F\right)
$$

where $K_{f}^{i}$ denotes the $i^{\text {th }}$ component of $K_{f}$. We recall that $K_{f}=V-\beta V^{G}$. Therefore, applying Hölder's inequality and using Lemma 2.4, we obtain

$$
\begin{aligned}
\left|I_{1}\right| \leq & \|F\|_{L^{2}} \sum_{i=1}^{2}\left\|(-\Delta)^{-\frac{3}{4}} \partial_{i}\left(K_{f}^{i}(-\Delta)^{\frac{3}{4}} F\right)\right\|_{L^{2}} \\
& +\bar{\alpha} e^{-\tau}\|F\|_{L^{2}} \sum_{i=1}^{2}\left\|(-\Delta)^{-\frac{3}{4}} \partial_{i}\left(K_{f}^{i}(-\Delta)^{\frac{7}{4}} F\right)\right\|_{L^{2}} \\
\leq & \|F\|_{L^{2}} \sum_{i=1}^{2}\left\|(-\Delta)^{-\frac{1}{4}}\left(K_{f}^{i}(-\Delta)^{\frac{3}{4}} F\right)\right\|_{L^{2}} \\
& +\bar{\alpha} e^{-\tau}\|F\|_{L^{2}} \sum_{i=1}^{2}\left\|(-\Delta)^{-\frac{1}{4}}\left(K_{f}^{i}(-\Delta)^{\frac{7}{4}} F\right)\right\|_{L^{2}} \\
\leq & C\|F\|_{L^{2}} \sum_{i=1}^{2}\left(\left\|K_{f}^{i}(-\Delta)^{\frac{3}{4}} F\right\|_{L^{2}(1)}+\bar{\alpha} e^{-\tau}\left\|K_{f}^{i}(-\Delta)^{\frac{7}{4}} F\right\|_{L^{2}(1)}\right) \\
\leq & C\|F\|_{L^{2}}\left\|K_{f}\right\|_{L^{\infty}}\left(\|f\|_{L^{2}(1)}+\bar{\alpha} e^{-\tau}\|\Delta f\|_{L^{2}(1)}\right)
\end{aligned}
$$


Using Lemma 2.2, part $(a)$, we obtain

$$
\left|I_{1}\right| \leq C\|F\|_{L^{2}}\|f\|_{H^{1}}^{\frac{1}{2}}\|f\|_{L^{2}(2)}^{\frac{1}{2}}\left(\|f\|_{L^{2}(1)}+\bar{\alpha} e^{-\tau}\|\Delta f\|_{L^{2}(1)}\right)
$$

Using the assumption on the bound (4.1) of $f$, we get

$$
\left|I_{1}\right| \leq C \sqrt{\delta}\|F\|_{L^{2}}\left(\|f\|_{L^{2}(1)}+\bar{\alpha} e^{-\tau}\|\Delta f\|_{L^{2}(1)}\right)
$$

The terms $I_{2}$ and $I_{3}$ are estimated in the same way as above and we get

$$
\begin{aligned}
\left|I_{2}\right| & \leq C|\beta|\|F\|_{L^{2}}\|f\|_{H^{1}}^{\frac{1}{2}}\|f\|_{L^{2}(2)}^{\frac{1}{2}}\left(\|G\|_{L^{2}(1)}+\bar{\alpha} e^{-\tau}\|\Delta G\|_{L^{2}(1)}\right) \\
& \leq C|\beta|\left(1+\bar{\alpha} e^{-\tau}\right)\|F\|_{L^{2}}\left(\|f\|_{H^{1}}+\|f\|_{L^{2}(2)}\right)
\end{aligned}
$$

and

$$
\begin{aligned}
\left|I_{3}\right| & \leq C|\beta|\|F\|_{L^{2}}\left\|V^{G}\right\|_{L^{\infty}}\left(\|f\|_{L^{2}(1)}+\bar{\alpha} e^{-\tau}\|\Delta f\|_{L^{2}(1)}\right) \\
& \leq C|\beta|\|F\|_{L^{2}}\left(\|f\|_{L^{2}(1)}+\bar{\alpha} e^{-\tau}\|\Delta f\|_{L^{2}(1)}\right)
\end{aligned}
$$

Collecting the bounds (4.8) to (4.14), we deduce from (4.4) that

$$
\begin{aligned}
& \partial_{\tau} E_{0}(\tau)+\frac{1}{4}\|F\|_{L^{2}}^{2}+\left(1+\frac{3 \bar{\alpha}}{4} e^{-\tau}\right)\|\nabla F\|_{L^{2}}^{2}+\bar{\epsilon} e^{-2 \tau}\|\Delta F\|_{L^{2}}^{2} \\
& \leq C|\beta| \bar{\alpha} e^{-\tau}\|F\|_{L^{2}}+C|\beta| \bar{\epsilon} e^{-2 \tau}\|\Delta F\|_{L^{2}} \\
&+C(\sqrt{\delta}+|\beta|)\|F\|_{L^{2}}\left(\|f\|_{L^{2}(1)}+\bar{\alpha} e^{-\tau}\|\Delta f\|_{L^{2}(1)}\right) \\
&+C|\beta|\left(1+\bar{\alpha} e^{-\tau}\right)\|F\|_{L^{2}}\left(\|f\|_{H^{1}}+\|f\|_{L^{2}(2)}\right)
\end{aligned}
$$

Applying Young's inequality yields that for all $\mu_{0}>0$, there exists a constant $C_{\mu_{0}}>0$ such that

$$
\begin{aligned}
\partial_{\tau} E_{0}(\tau) & +\left(\frac{1}{4}-\mu_{0}\right)\|F\|_{L^{2}}^{2}+\left(1+\frac{3 \bar{\alpha}}{4} e^{-\tau}\right)\|\nabla F\|_{L^{2}}^{2}+\bar{\epsilon} e^{-2 \tau}\left(1-\mu_{0}\right)\|\Delta F\|_{L^{2}}^{2} \\
\leq & C_{\mu_{0}}|\beta|^{2}\left(\bar{\alpha}^{2}+\bar{\epsilon}\right) e^{-2 \tau} \\
+ & C_{\mu_{0}}\left(\delta+|\beta|^{2}\right)\left(\|f\|_{L^{2}(1)}^{2}+\bar{\alpha}^{2} e^{-2 \tau}\|\Delta f\|_{L^{2}(1)}^{2}\right) \\
& +C_{\mu_{0}}|\beta|^{2}\left(1+\bar{\alpha}^{2} e^{-2 \tau}\right)\left(\|f\|_{H^{1}}^{2}+\|f\|_{L^{2}(2)}^{2}\right)
\end{aligned}
$$

Remarking that

$$
\begin{aligned}
\|f\|_{L^{2}(1)}^{2} & =\int_{\mathbb{R}^{2}}\left(1+|\xi|^{2}\right)|f(\xi)|^{2} d \xi \\
& \leq\|f\|_{L^{2}}\|f\|_{L^{2}(2)} \leq \frac{1}{2}\left(\|f\|_{L^{2}}^{2}+\left\||\xi|^{2} f\right\|_{L^{2}}^{2}\right) .
\end{aligned}
$$


Using (4.16) and the fact that $f$ satisfies the bound (4.1), we deduce form (4.15) that

$$
\begin{aligned}
\partial_{\tau} E_{0}(\tau)+ & \left(\frac{1}{4}-\mu_{0}\right)\|F\|_{L^{2}}^{2}+\left(1+\frac{3 \bar{\alpha}}{4} e^{-\tau}\right)\|\nabla F\|_{L^{2}}^{2}+\bar{\epsilon} e^{-2 \tau}\left(1-\mu_{0}\right)\|\Delta F\|_{L^{2}}^{2} \\
\leq & C_{\mu_{0}}|\beta|^{2}\left(\bar{\alpha}^{2}+\bar{\epsilon}\right) e^{-2 \tau}+C_{\mu_{0}}|\beta|^{2}\|\nabla f\|_{L^{2}}^{2} \\
& +C_{\mu_{0}}\left(\delta+|\beta|^{2}\right)\left(\|f\|_{L^{2}}^{2}+\left\||\xi|^{2} f\right\|_{L^{2}}^{2}\right) \\
& +C_{\mu_{0}}\left(\delta+|\beta|^{2}\right) \bar{\alpha}^{2} e^{-2 \tau}\left(\|\Delta f\|_{L^{2}}^{2}+\left\||\xi|^{2} \Delta f\right\|_{L^{2}}^{2}\right)
\end{aligned}
$$

Now, let $0<\mu_{0}<\frac{1}{4}$ and $\frac{\theta}{2}=\frac{1}{4}-\mu_{0}<\frac{1}{4}$. The above inequality becomes

$$
\begin{aligned}
\partial_{\tau} E_{0}(\tau)+ & \theta E_{0}(\tau)+\left(1+\frac{3 \bar{\alpha}}{4} e^{-\tau}-\frac{\theta \bar{\alpha}}{2} e^{-\tau}\right)\|\nabla F\|_{L^{2}}^{2}+\bar{\epsilon}\left(\frac{3}{4}+\frac{\theta}{2}\right) e^{-2 \tau}\|\Delta F\|_{L^{2}}^{2} \\
\leq & C_{0}|\beta|^{2}\left(\bar{\alpha}^{2}+\bar{\epsilon}\right) e^{-2 \tau}+C_{0}|\beta|^{2}\|\nabla f\|_{L^{2}}^{2} \\
& +C_{0}\left(\delta+|\beta|^{2}\right)\left(\|f\|_{L^{2}}^{2}+\left\||\xi|^{2} f\right\|_{L^{2}}^{2}\right) \\
& +C_{0}\left(\delta+|\beta|^{2}\right) \bar{\alpha}^{2} e^{-2 \tau}\left(\|\Delta f\|_{L^{2}}^{2}+\left\||\xi|^{2} \Delta f\right\|_{L^{2}}^{2}\right)
\end{aligned}
$$

Next, we will give estimates of $f$ and $\Delta f$.

4.1.2. Estimates of $f$ and $\nabla f$. In order to estimate $\|f\|_{L^{2}}$ and $\|\nabla f\|_{L^{2}}$, we introduce the functional $E_{1}(\tau)$ given by

$$
E_{1}(\tau)=\frac{1}{2}\left(\|f(\tau)\|_{L^{2}}^{2}+\bar{\alpha} e^{-\tau}\|\nabla f(\tau)\|_{L^{2}}^{2}\right)
$$

We have the following lemma.

Lemma 4.2. Assume that $f \in L^{\infty}\left(\left[\tau_{0}, \tau_{1}\right], H^{2}(2)\right)$ is a solution of (3.14) satisfying the bound (4.1). Then, there exists a positive constant $C_{1}$ such that, for all $\tau \in\left[\tau_{0}, \tau_{1}\right]$,

$$
\begin{gathered}
\partial_{\tau} E_{1}(\tau)+E_{1}(\tau)+\left(\frac{1}{6}-\frac{\bar{\alpha}}{2} e^{-\tau}\right)\|\nabla f\|_{L^{2}}^{2}+\frac{\bar{\epsilon}}{2} e^{-2 \tau}\|\Delta f\|_{L^{2}}^{2} \\
\leq C_{1}|\beta|^{2}\left(\bar{\alpha}^{2}+\bar{\epsilon}\right) e^{-2 \tau}+\left(\frac{2}{3}+C_{1}|\beta|^{2}\right)\|\nabla F\|_{L^{2}}^{2} \\
\quad+C_{1}|\beta|^{2}\left\||\xi|^{2} f\right\|_{L^{2}}^{2}+C_{1}\left(\delta+|\beta|^{2}\right) \bar{\alpha}^{2} e^{-2 \tau}\|\Delta f\|_{L^{2}}^{2}
\end{gathered}
$$

Proof : Taking the scalar product in $L^{2}\left(\mathbb{R}^{2}\right)$ of (3.14) with $f$, we obtain

$$
\begin{aligned}
& \partial_{\tau} E_{1}(\tau)+\|\nabla f\|_{L^{2}}^{2}-\frac{1}{2}\|f\|_{L^{2}}^{2}+\bar{\epsilon} e^{-2 \tau}\|\Delta f\|_{L^{2}}^{2} \\
& +\beta \frac{\bar{\alpha}}{2} e^{-\tau}(\xi . \nabla \Delta G, f)+\beta \bar{\epsilon} e^{-2 \tau}\left(\Delta^{2} G, f\right)+\beta \bar{\alpha} e^{-\tau}(\Delta G, f) \\
& +J_{1}+\beta J_{2}+\beta J_{3}=0
\end{aligned}
$$


where

$$
\begin{aligned}
& J_{1}=\left(K_{f} \cdot \nabla\left(f-\bar{\alpha} e^{-\tau} \Delta f\right), f\right) \\
& J_{2}=\left(V^{G} \cdot \nabla\left(f-\bar{\alpha} e^{-\tau} \Delta f\right), f\right) \\
& J_{3}=\left(K_{f} \cdot \nabla\left(G-\bar{\alpha} e^{-\tau} \Delta G\right), f\right) .
\end{aligned}
$$

Integrating by parts and applying the Hölder inequality, we have the following bounds

$$
|(\xi . \nabla \Delta G, f)| \leq\left(2\|\Delta G\|_{L^{2}}+\||\xi| \Delta G\|_{L^{2}}\right)\|\nabla f\|_{L^{2}} \leq C\|\nabla f\|_{L^{2}}
$$

$$
\begin{aligned}
\left|\left(\Delta^{2} G, f\right)\right| & \leq\|\Delta G\|_{L^{2}}\|\Delta f\|_{L^{2}} \leq C\|\Delta f\|_{L^{2}} \\
|(\Delta G, f)| & \leq\|\nabla G\|_{L^{2}}\|\nabla f\|_{L^{2}} \leq C\|\nabla f\|_{L^{2}}
\end{aligned}
$$

Next, we bound the terms $J_{i}, i=1,2,3$. Integrating by parts and using the fact that $\operatorname{div} K_{f}=0$, we can write $J_{1}$ as

$$
J_{1}=-\bar{\alpha} e^{-\tau}\left(K_{f} \cdot \nabla f, \Delta f\right)
$$

Thus, applying Hölder's inequality and using Lemma 2.2 part (a), we obtain the following bound

$$
\begin{aligned}
\left|J_{1}\right| & \leq \bar{\alpha} e^{-\tau}\left\|K_{f}\right\|_{L^{\infty}}\|\nabla f\|_{L^{2}}\|\Delta f\|_{L^{2}} \\
& \leq C \bar{\alpha} e^{-\tau}\|f\|_{H^{1}}^{\frac{1}{2}}\|f\|_{L^{2}(2)}^{\frac{1}{2}}\|\nabla f\|_{L^{2}}\|\Delta f\|_{L^{2}} \leq C \sqrt{\delta} \bar{\alpha} e^{-\tau}\|\nabla f\|_{L^{2}}\|\Delta f\|_{L^{2}}
\end{aligned}
$$

since $f$ satisfies the bound (4.1).

On the other hand, since $\operatorname{div} V^{G}=0$, the term $J_{2}$ can be estimated as above and we have

$$
\left|J_{2}\right| \leq \bar{\alpha} e^{-\tau}\left\|V^{G}\right\|_{L^{\infty}}\|\nabla f\|_{L^{2}}\|\Delta f\|_{L^{2}} \leq C \bar{\alpha} e^{-\tau}\|\nabla f\|_{L^{2}}\|\Delta f\|_{L^{2}}
$$

Finally, integrating by parts, using Lemma 2.2 part (b), we obtain the following bound on $J_{3}$

$$
\left|J_{3}\right| \leq\left\|K_{f}\right\|_{L^{4}}\left\|G-\bar{\alpha} e^{-\tau} \Delta G\right\|_{L^{4}}\|\nabla f\|_{L^{2}} \leq C\left(1+\bar{\alpha} e^{-\tau}\right)\|f\|_{L^{2}(2)}\|\nabla f\|_{L^{2}}
$$

Collecting the bounds (4.19) to (4.24) and applying Young's inequality on (4.18), we show that, for all $\mu_{1}>0$, there exists a constant $C_{\mu_{1}}>0$ such that

$$
\begin{aligned}
& \partial_{\tau} E_{1}(\tau)+\left(1-\mu_{1}\right)\|\nabla f\|_{L^{2}}^{2}-\frac{1}{2}\|f\|_{L^{2}}^{2}+\bar{\epsilon} e^{-2 \tau}\left(1-\mu_{1}\right)\|\Delta f\|_{L^{2}}^{2} \\
& \leq C_{\mu_{1}}|\beta|^{2}\left(\bar{\alpha}^{2}+\bar{\epsilon}\right) e^{-2 \tau}+C_{\mu_{1}}|\beta|^{2}\left(1+\bar{\alpha}^{2} e^{-2 \tau}\right)\|f\|_{L^{2}(2)}^{2} \\
& \quad+C_{\mu_{1}}\left(\delta+|\beta|^{2}\right) \bar{\alpha}^{2} e^{-2 \tau}\|\Delta f\|_{L^{2}}^{2}
\end{aligned}
$$

Choosing $\mu_{1}=\frac{1}{2}$ and remarking that

$$
\|f\|_{L^{2}(2)}^{2} \leq 2\left(\|f\|_{L^{2}}^{2}+\left\||\xi|^{2} f\right\|_{L^{2}}^{2}\right)
$$


we deduce from the above inequality that $(4.26)$

$$
\begin{aligned}
& \partial_{\tau} E_{1}(\tau)+E_{1}(\tau)+\left(\frac{1}{2}-\frac{\bar{\alpha}}{2} e^{-\tau}\right)\|\nabla f\|_{L^{2}}^{2}+\frac{\bar{\epsilon}}{2} e^{-2 \tau}\|\Delta f\|_{L^{2}}^{2} \\
& \quad \leq C_{1}|\beta|^{2}\left(\bar{\alpha}^{2}+\bar{\epsilon}\right) e^{-2 \tau}+\left(1+C_{1}|\beta|^{2}\left(1+\bar{\alpha}^{2} e^{-2 \tau}\right)\right)\|f\|_{L^{2}}^{2} \\
& \quad+C_{1}|\beta|^{2}\left(1+\bar{\alpha}^{2} e^{-2 \tau}\right)\left\||\xi|^{2} f\right\|_{L^{2}}^{2}+C_{1}\left(\delta+|\beta|^{2}\right) \bar{\alpha}^{2} e^{-2 \tau}\|\Delta f\|_{L^{2}}^{2}
\end{aligned}
$$

Using (3.19), we can write Inequality (4.26) as

$$
\begin{gathered}
\partial_{\tau} E_{1}(\tau)+E_{1}(\tau)+\left(\frac{1}{6}-\frac{\bar{\alpha}}{2} e^{-\tau}\right)\|\nabla f\|_{L^{2}}^{2}+\frac{\bar{\epsilon}}{2} e^{-2 \tau}\|\Delta f\|_{L^{2}}^{2} \\
\leq C_{1}|\beta|^{2}\left(\bar{\alpha}^{2}+\bar{\epsilon}\right) e^{-2 \tau}+\left(\frac{2}{3}+C_{1}|\beta|^{2}\right)\|\nabla F\|_{L^{2}}^{2} \\
\quad+C_{1}|\beta|^{2}\left\||\xi|^{2} f\right\|_{L^{2}}^{2}+C_{1}\left(\delta+|\beta|^{2}\right) \bar{\alpha}^{2} e^{-2 \tau}\|\Delta f\|_{L^{2}}^{2} .
\end{gathered}
$$

4.1.3. Estimates of $\nabla f$ and $\Delta f$. In order to estimate $\|\nabla f\|_{L^{2}}$ and $\|\Delta f\|_{L^{2}}$, we introduce the functional is

$$
E_{2}(\tau)=\frac{1}{2}\left(\|\nabla f(\tau)\|_{L^{2}}^{2}+\bar{\alpha} e^{-\tau}\|\Delta f(\tau)\|_{L^{2}}^{2}\right)
$$

LEMMA 4.3. Assume that $f \in L^{\infty}\left(\left[\tau_{0}, \tau_{1}\right], H^{2}(2)\right)$ is a solution of (3.14) satisfying the bound (4.1). Then, there exists a positive constant $C_{2}$ such that for all $\tau \in\left[\tau_{0}, \tau_{1}\right]$, we have

$$
\begin{aligned}
& \partial_{\tau} E_{2}(\tau)+E_{2}(\tau)+\left(\frac{1}{2}-\bar{\alpha} e^{-\tau}\right)\|\Delta f\|_{L^{2}}^{2}+\frac{\bar{\epsilon}}{2} e^{-2 \tau}\|\nabla \Delta f\|_{L^{2}}^{2} \\
& \leq C_{2}|\beta|^{2}\left(\bar{\alpha}^{2}+\bar{\epsilon}\right) e^{-2 \tau}+\left(\frac{3}{2}+C_{2}\left(\delta+|\beta|^{2}\right)\right)\|\nabla f\|_{L^{2}}^{2} \\
& \quad+C_{2}|\beta|^{2}\left(\left\||\xi|^{2} f\right\|_{L^{2}}^{2}+\|f\|_{L^{2}}^{2}\right)
\end{aligned}
$$

Proof : In order to obtain estimates of the $L^{2}$-norm of $\Delta f$, we take the scalar product in $L^{2}\left(\mathbb{R}^{2}\right)$ of $(3.14)$ with $-\Delta f$. We obtain

$$
\begin{gathered}
\partial_{\tau} E_{2}(\tau)+(\mathcal{L} f, \Delta f)+\bar{\epsilon} e^{-2 \tau}\|\nabla \Delta f\|_{L^{2}}^{2}-\frac{\bar{\alpha}}{2} e^{-\tau}\|\Delta f\|_{L^{2}}^{2} \\
\leq|\beta| \bar{\alpha} e^{-\tau}\left(\frac{1}{2}\||\xi| \nabla \Delta G\|_{L^{2}}+\|\Delta G\|_{L^{2}}\right)\|\Delta f\|_{L^{2}} \\
\quad+\bar{\epsilon} \beta e^{-2 \tau}\|\Delta G\|_{L^{2}}\|\Delta f\|_{L^{2}}+K_{1}+\beta K_{2}+\beta K_{3}
\end{gathered}
$$

where

$$
\begin{aligned}
& K_{1}=\left(K_{f} \cdot \nabla\left(f-\bar{\alpha} e^{-\tau} \Delta f\right), \Delta f\right) \\
& K_{2}=\left(V^{G} \cdot \nabla\left(f-\bar{\alpha} e^{-\tau} \Delta f\right), \Delta f\right) \\
& K_{3}=\left(K_{f} \cdot \nabla\left(G-\bar{\alpha} e^{-\tau} \Delta G\right), \Delta f\right)
\end{aligned}
$$

A simple integration by parts implies that $(\xi . \nabla f, \Delta f)=0$. Thus,

$$
(\mathcal{L} f, \Delta f)=\|\Delta f\|_{L^{2}}^{2}-\|\nabla f\|_{L^{2}}^{2} .
$$


Since $\operatorname{div} V^{G}=\operatorname{div} K_{f}=0$, we remark that

$$
\left(K_{f} . \nabla \Delta f, \Delta f\right)=\left(V^{G} \cdot \nabla \Delta f, \Delta f\right)=0 .
$$

Therefore, using Lemma 2.2 part (a), we can write

$$
\begin{aligned}
\left|K_{1}\right| & \leq\left\|K_{f}\right\|_{L^{\infty}}\|\nabla f\|_{L^{2}}\|\Delta f\|_{L^{2}} \leq C\|f\|_{H^{1}}^{\frac{1}{2}}\|f\|_{L^{2}(2)}^{\frac{1}{2}}\|\nabla f\|_{L^{2}}\|\Delta f\|_{L^{2}} \\
& \leq C \sqrt{\delta}\|\nabla f\|_{L^{2}}\|\Delta f\|_{L^{2}} .
\end{aligned}
$$

We also have

$$
\left|K_{2}\right| \leq\left\|V^{G}\right\|_{L^{\infty}}\|\nabla f\|_{L^{2}}\|\Delta f\|_{L^{2}} \leq C\|\nabla f\|_{L^{2}}\|\Delta f\|_{L^{2}}
$$

Finally, using Lemma 2.2 part (b), we can write

$$
\left|K_{3}\right| \leq\left\|K_{f}\right\|_{L^{4}}\left\|\nabla\left(G-\bar{\alpha} e^{-\tau} \Delta G\right)\right\|_{L^{4}}\|\Delta f\|_{L^{2}} \leq C\left(1+\bar{\alpha} e^{-\tau}\right)\|f\|_{L^{2}(2)}\|\Delta f\|_{L^{2}}
$$

Thus, using the bounds (4.30), (4.31) and (4.32) and applying the Cauchy-Schwarz inequality to the estimate (4.29), we obtain that, for all $\mu_{2}>0$, there exists a constant $C_{\mu_{2}}>0$ such that

$$
\begin{aligned}
& \partial_{\tau} E_{2}(\tau)+\left(1-\frac{\bar{\alpha}}{2} e^{-\tau}-\mu_{2}\right)\|\Delta f\|_{L^{2}}^{2}-\|\nabla f\|_{L^{2}}^{2}+\bar{\epsilon} e^{-2 \tau}\left(1-\mu_{2}\right)\|\nabla \Delta f\|_{L^{2}}^{2} \\
& \quad \leq C_{\mu_{2}}|\beta|^{2}\left(\bar{\alpha}^{2}+\bar{\epsilon}\right) e^{-2 \tau}+C_{\mu_{2}}\left(\delta+|\beta|^{2}\right)\|\nabla f\|_{L^{2}}^{2} \\
& \quad+C_{\mu_{2}}|\beta|^{2}\left(\left\||\xi|^{2} f\right\|_{L^{2}}^{2}+\|f\|_{L^{2}}^{2}\right)
\end{aligned}
$$

Choosing $\mu_{2}=\frac{1}{2}$, we get

$$
\begin{gathered}
\partial_{\tau} E_{2}(\tau)+E_{2}(\tau)+\left(\frac{1}{2}-\bar{\alpha} e^{-\tau}\right)\|\Delta f\|_{L^{2}}^{2}+\frac{\bar{\epsilon}}{2} e^{-2 \tau}\|\nabla \Delta f\|_{L^{2}}^{2} \\
\leq C_{2}|\beta|^{2}\left(\bar{\alpha}^{2}+\bar{\epsilon}\right) e^{-2 \tau}+\left(\frac{3}{2}+C_{2}\left(\delta+|\beta|^{2}\right)\right)\|\nabla f\|_{L^{2}}^{2} \\
\quad+C_{2}|\beta|^{2}\left(\left\||\xi|^{2} f\right\|_{L^{2}}^{2}+\|f\|_{L^{2}}^{2}\right)
\end{gathered}
$$

Thus, Lemma 4.3 is proved.

In order to obtain an estimate of $\|f\|_{H^{1}}^{2}+\bar{\alpha} e^{-\tau}\|\Delta f\|_{L^{2}}^{2}$, we introduce the functional $E_{3}$ given by

$$
E_{3}(\tau)=5 E_{0}(\tau)+5 E_{1}(\tau)+\frac{1}{2} E_{2}(\tau)
$$

Indeed, we have the following inequality

$$
\|f\|_{H^{1}}^{2}+\bar{\alpha} e^{-\tau}\|\Delta f\|_{L^{2}}^{2} \leq C E_{3}(\tau)
$$

where $C>0$ is a constant independent of $\bar{\alpha}$.

Lemmas 4.1, 4.2, 4.3 and Inequality (3.19) imply that there exists a constant $C_{3}>0$ 
such that for all $\tau \in\left[\tau_{0}, \tau_{1}\right]$, we have

$$
\begin{gathered}
\partial_{\tau} E_{3}(\tau)+\theta E_{3}(\tau)+\bar{\epsilon} e^{-\tau}\left(\left(\frac{15}{4}+\frac{5 \theta}{2}\right)\|\Delta F\|_{L^{2}}^{2}+\frac{5}{2}\|\Delta f\|_{L^{2}}^{2}+\frac{1}{4}\|\nabla \Delta f\|_{L^{2}}^{2}\right) \\
+\left(\frac{5}{3}+\frac{5 \bar{\alpha}}{4} e^{-\tau}-\frac{5 \theta \bar{\alpha}}{2}-C_{3}\left(|\beta|^{2}+\delta\right)\right)\|\nabla F\|_{L^{2}}^{2} \\
+\left(\frac{1}{12}-\frac{5 \bar{\alpha}}{2}-C_{3}\left(|\beta|^{2}+\delta\right)\right)\|\nabla f\|_{L^{2}}^{2}+\left(\frac{1}{4}-\frac{\bar{\alpha}}{2} e^{-\tau}-C_{3} \bar{\alpha}^{2}\left(|\beta|^{2}+\delta\right)\right)\|\Delta f\|_{L^{2}}^{2} \\
\leq C_{3}|\beta|^{2}\left(\bar{\alpha}^{2}+\bar{\epsilon}\right) e^{-\tau}+C_{3}\left(|\beta|^{2}+\delta\right)\left\||\xi|^{2} f\right\|_{L^{2}}^{2} \\
+C_{3}\left(|\beta|^{2}+\delta\right) \bar{\alpha}^{2} e^{-2 \tau}\left\||\xi|^{2} \Delta f\right\|_{L^{2}}^{2}
\end{gathered}
$$

where $0<\theta<\frac{1}{2}$.

4.2. Energy estimates in $L^{2}(2)$. In order to bound the $H^{2}$ weighted norm of $f$, it remains to estimate the term $\||\xi|^{2}\left(f(\tau)-\bar{\alpha} e^{-\tau} \Delta f(\tau) \|_{L^{2}}\right.$. For this purpose, we introduce the weighted functional

$$
E_{4}(\tau)=\frac{1}{2} \||\xi|^{2}\left(f(\tau)-\bar{\alpha} e^{-\tau} \Delta f(\tau) \|_{L^{2}}^{2}\right.
$$

We have the following lemma.

Lemma 4.4. Assume that $f \in L^{\infty}\left(\left[\tau_{0}, \tau_{1}\right], H^{2}(2)\right)$ is a solution of (3.14) satisfying the bound (4.1). Then, there exists a constant $C_{4}>0$ such that for all $\tau \in\left[\tau_{0}, \tau_{1}\right]$,

$$
\begin{aligned}
& \partial_{\tau} E_{4}(\tau)+\frac{1}{2} E_{4}(\tau)+\left(\frac{1}{12}-C_{4}\left(\sqrt{\delta}+\bar{\alpha} e^{-\tau}+\delta\right)\right)\left\||\xi|^{2} f\right\|_{L^{2}}^{2} \\
& +\left(1+\frac{\bar{\alpha}}{2} e^{-\tau}-\bar{\epsilon} e^{-2 \tau} C_{4}\right)\left\||\xi|^{2} \nabla f\right\|_{L^{2}}^{2} \\
& +\bar{\alpha} e^{-\tau}\left(1+\frac{\bar{\alpha}}{4} e^{-\tau}-\bar{\alpha} e^{-\tau}\left(\sqrt{\delta}+\frac{1}{12}+C_{4} \bar{\epsilon} e^{-2 \tau}\right)\right)\left\||\xi|^{2} \Delta f\right\|_{L^{2}}^{2} \\
& +\bar{\epsilon} e^{-2 \tau}\left(\left\||\xi|^{2} \Delta f\right\|_{L^{2}}^{2}+32\|f\|_{L^{2}}^{2}+\bar{\alpha} e^{-\tau}\left\||\xi|^{2} \nabla \Delta f\right\|_{L^{2}}^{2}\right) \\
& \leq C_{4} \delta\left(\bar{\epsilon}^{2}+\bar{\alpha}^{2}\right) e^{-2 \tau}+\left(48+C_{4}\left(\bar{\alpha} e^{-\tau}+\sqrt{\delta}\right)\|f\|_{L^{2}}^{2}\right. \\
& +C_{4} \bar{\epsilon} e^{-2 \tau}\|\nabla f\|_{L^{2}}^{2}+\left(C_{4} \bar{\epsilon} \bar{\alpha} e^{-3 \tau}+\bar{\alpha}^{2} e^{-2 \tau} C_{4} \sqrt{\delta}\right)\|\Delta f\|_{L^{2}}^{2}
\end{aligned}
$$

Proof : In order to estimate $E_{4}(\tau)$, we take the scalar product of Equation (3.14) with $|\xi|^{4}\left(f-\bar{\alpha} e^{-\tau} \Delta f\right)$. 
We obtain

(4.36)

$$
\begin{gathered}
\partial_{\tau} E_{4}(\tau)-\left(\mathcal{L} f,|\xi|^{4}\left(f-\bar{\alpha} e^{-\tau} \Delta f\right)\right)+\bar{\epsilon} e^{-2 \tau}\left(\Delta^{2} f,|\xi|^{4}\left(f-\bar{\alpha} e^{-\tau} \Delta f\right)\right) \\
+\frac{\bar{\alpha}}{2} e^{-\tau}\left(\xi . \nabla \Delta f,|\xi|^{4}\left(f-\bar{\alpha} e^{-\tau} \Delta f\right)\right)+\bar{\alpha} e^{-\tau}\left(\Delta f,|\xi|^{4}\left(f-\bar{\alpha} e^{-\tau} \Delta f\right)\right) \\
\leq\left|M_{1}\right|+\left|M_{2}\right|+\left|M_{3}\right| \\
+|\beta| e^{-\tau}|||\xi|^{2}\left(f-\bar{\alpha} e^{-\tau} \Delta f\right) \|_{L^{2}}\left(\bar{\epsilon} e^{-\tau}\left\||\xi|^{2} \Delta^{2} G\right\|_{L^{2}}+\bar{\alpha}\left\||\xi|^{2} \Delta G\right\|_{L^{2}}\right. \\
\left.+\frac{\bar{\alpha}}{2}\left\||\xi|^{3} \nabla \Delta G\right\|_{L^{2}}\right)
\end{gathered}
$$

where

$$
\begin{aligned}
& M_{1}=\beta\left(V^{G} \cdot \nabla\left(f-\bar{\alpha} e^{-\tau} \Delta f\right),|\xi|^{4}\left(f-\bar{\alpha} e^{-\tau} \Delta f\right),\right. \\
& M_{2}=\beta\left(K_{f} \cdot \nabla\left(G-\bar{\alpha} e^{-\tau} \Delta G\right),|\xi|^{4}\left(f-\bar{\alpha} e^{-\tau} \Delta f\right)\right), \\
& M_{3}=\left(K_{f} \cdot \nabla\left(f-\bar{\alpha} e^{-\tau} \Delta f\right),|\xi|^{4}\left(f-\bar{\alpha} e^{-\tau} \Delta f\right)\right) .
\end{aligned}
$$

We begin by computing the term $\left(\mathcal{L} f,|\xi|^{4}\left(f-\bar{\alpha} e^{-\tau} \Delta f\right)\right)$. Using an integrating by parts, we obtain

$$
\begin{aligned}
\left(\Delta f,|\xi|^{4} f\right) & =-\left\||\xi|^{2} \nabla f\right\|_{L^{2}}^{2}+8\||\xi| f\|_{L^{2}}^{2} \\
\frac{1}{2}\left(\xi . \nabla f,|\xi|^{4} f\right) & =-\frac{3}{2}\left\||\xi|^{2} f\right\|_{L^{2}}^{2}
\end{aligned}
$$

Thus,

$$
-\left(\mathcal{L} f,|\xi|^{4} f\right)=\left\||\xi|^{2} \nabla f\right\|_{L^{2}}^{2}-8\||\xi| f\|_{L^{2}}^{2}+\frac{1}{2}\left\||\xi|^{2} f\right\|_{L^{2}}^{2} .
$$

Next, we compute the term $\frac{\alpha}{\nu} e^{-\tau}\left(\mathcal{L} f,|\xi|^{4} \Delta f\right)$. Integrating by parts yields

$$
\begin{gathered}
\frac{1}{2}\left(\xi . \nabla f,|\xi|^{4} \Delta f\right)=-\frac{1}{2}\left(\xi . \nabla \Delta f,|\xi|^{4} f\right)-3\left(f,|\xi|^{4} \Delta f\right) \\
=-\frac{1}{2}\left(\xi . \nabla \Delta f,|\xi|^{4} f\right)+3\left\||\xi|^{2} \nabla f\right\|_{L^{2}}^{2}-24\||\xi| f\|_{L^{2}}^{2}
\end{gathered}
$$

Thus, using the equalities (4.37) and (4.39), we get

$$
\begin{aligned}
& \bar{\alpha} e^{-\tau}\left(\mathcal{L} f,|\xi|^{4} \Delta f\right)=\bar{\alpha} e^{-\tau}\left(\left\||\xi|^{2} \Delta f\right\|_{L^{2}}^{2}+2\left\||\xi|^{2} \nabla f\right\|_{L^{2}}^{2}\right. \\
& \left.\quad-16\||\xi| f\|_{L^{2}}^{2}-\frac{1}{2}\left(\xi . \nabla \Delta f,|\xi|^{4} f\right)\right)
\end{aligned}
$$

Equalities (4.38) and (4.40) imply that

$$
\begin{aligned}
& -\left(\mathcal{L} f,|\xi|^{4}\left(f-\bar{\alpha} e^{-\tau} \Delta f\right)\right)= \\
& -\frac{\bar{\alpha}}{2} e^{-\tau}\left(\xi . \nabla \Delta f,|\xi|^{4} f\right)-8\left(1+2 \bar{\alpha} e^{-\tau}\right)\||\xi| f\|_{L^{2}}^{2} \\
& \quad+\frac{1}{2}\left\||\xi|^{2} f\right\|_{L^{2}}^{2}+\left(1+2 \bar{\alpha} e^{-\tau}\right)\left\||\xi|^{2} \nabla f\right\|_{L^{2}}^{2}+\bar{\alpha} e^{-\tau}\left\||\xi|^{2} \Delta f\right\|_{L^{2}}^{2}
\end{aligned}
$$

On the other hand,

$$
-\bar{\alpha}^{2} e^{-2 \tau}\left(\frac{1}{2} \xi . \nabla \Delta f+\Delta f,|\xi|^{4} \Delta f\right)=\frac{\bar{\alpha}^{2}}{2} e^{-2 \tau}\left\||\xi|^{2} \Delta f\right\|_{L^{2}}^{2}
$$


and

$$
\begin{aligned}
& \bar{\epsilon} e^{-2 \tau}\left(\Delta^{2} f,|\xi|^{4} f\right) \\
& =\bar{\epsilon} e^{-2 \tau}\left(\left\||\xi|^{2} \Delta f\right\|_{L^{2}}^{2}+16\left(\Delta f,|\xi|^{2} f\right)+8\left(\xi . \nabla f,|\xi|^{2} \Delta f\right)\right) \\
& =\frac{\epsilon}{\nu^{2}} e^{-2 \tau}\left(\left\||\xi|^{2} \Delta f\right\|_{L^{2}}^{2}-16\||\xi| \nabla f\|_{L^{2}}^{2}+32\|f\|_{L^{2}}^{2}+8\left(\xi . \nabla f,|\xi|^{2} \Delta f\right)\right) .
\end{aligned}
$$

Again, integrating by parts, we obtain

$$
\begin{aligned}
8\left(\xi . \nabla f,|\xi|^{2} \Delta f\right) & =8\||\xi| \nabla f\|_{L^{2}}^{2}-16(\xi . \nabla f, \xi . \nabla f) \\
& \geq 8\||\xi| \nabla f\|_{L^{2}}^{2}-16\||\xi| \nabla f\|_{L^{2}}^{2}=-8\||\xi| \nabla f\|_{L^{2}}^{2} .
\end{aligned}
$$

Therefore,

$$
\bar{\epsilon} e^{-2 \tau}\left(\Delta^{2} f,|\xi|^{4} f\right) \geq \bar{\epsilon} e^{-2 \tau}\left(\left\||\xi|^{2} \Delta f\right\|_{L^{2}}^{2}-24\||\xi| \nabla f\|_{L^{2}}^{2}+32\|f\|_{L^{2}}^{2}\right) .
$$

Finally,

$$
-\bar{\epsilon} \bar{\alpha} e^{-3 \tau}\left(\Delta^{2} f,|\xi|^{4} \Delta f\right)=\bar{\epsilon} \bar{\alpha} e^{-3 \tau}\left(\left\||\xi|^{2} \nabla \Delta f\right\|_{L^{2}}^{2}-8\||\xi| \Delta f\|_{L^{2}}^{2}\right)
$$

Putting together the estimates (4.41), (4.42) and (4.43), we obtain the following inequality for the left-hand side of (4.36), that is,

$$
\begin{aligned}
& \partial_{\tau} E_{4}(\tau)+\frac{1}{2}\left\||\xi|^{2} f\right\|_{L^{2}}^{2}+\left(1+\bar{\alpha} e^{-\tau}\right)\left\||\xi|^{2} \nabla f\right\|_{L^{2}}^{2} \\
& +\bar{\alpha} e^{-\tau}\left(1+\frac{\bar{\alpha}}{2} e^{-\tau}\right)\left\||\xi|^{2} \Delta f\right\|_{L^{2}}^{2} \\
& +\bar{\epsilon} e^{-2 \tau}\left(\left\||\xi|^{2} \Delta f\right\|_{L^{2}}^{2}+32\|f\|_{L^{2}}^{2}+\bar{\alpha} e^{-\tau}\left\||\xi|^{2} \nabla \Delta f\right\|_{L^{2}}^{2}\right) \\
& -\left(8+8 \bar{\alpha} e^{-\tau}\right)\||\xi| f\|_{L^{2}}^{2}-\bar{\epsilon} e^{-2 \tau}\left(24\||\xi| \nabla f\|_{L^{2}}^{2}+8 \bar{\alpha} e^{-\tau}\||\xi| \Delta f\|_{L^{2}}^{2}\right) \\
& \leq\left|M_{1}\right|+\left|M_{2}\right|+\left|M_{3}\right| \\
& +|\beta| e^{-\tau}\left\||\xi|^{2}\left(f-\bar{\alpha} e^{-\tau} \Delta f\right)\right\|_{L^{2}}\left(\bar{\epsilon} e^{-\tau}\left\||\xi|^{2} \Delta^{2} G\right\|_{L^{2}}+\bar{\alpha}\left\||\xi|^{2} \Delta G\right\|_{L^{2}}\right. \\
& \left.+\frac{\bar{\alpha}}{2}\left\||\xi|^{3} \nabla \Delta G\right\|_{L^{2}}\right)
\end{aligned}
$$

It remains to bound the terms $M_{i} ; i=1,2,3$.

Integrating by parts and using the fact that $\operatorname{div} V_{G}=0$, we obtain

$$
M_{1}=-2 \int_{\mathbb{R}^{2}}\left(V_{G} \cdot \xi\right)|\xi|^{2}\left|f-\bar{\alpha} e^{-\tau} \Delta f\right|^{2} d \xi
$$

Using remark 3.1, we deduce that $M_{1}=0$.

In order to bound $M_{2}$, we use Lemma 2.2 part (b). We obtain

$$
\begin{aligned}
\left|M_{2}\right| & \leq|\beta|\left\|K_{f}\right\|_{L^{4}}\left\||\xi|^{2}\left(G-\bar{\alpha} e^{-\tau} \Delta G\right)\right\|_{L^{4}}\left\||\xi|^{2}\left(f-\bar{\alpha} e^{-\tau} \Delta f\right)\right\|_{L^{2}} \\
& \leq C|\beta|\|f\|_{L^{2}(2)}\left\||\xi|^{2}\left(f-\bar{\alpha} e^{-\tau} \Delta f\right)\right\|_{L^{2}}
\end{aligned}
$$


Then, the Cauchy-Schwarz inequality implies that for all $\lambda>0$, there exists a constant $C_{\lambda}>0$ such that

$$
\begin{aligned}
\left|M_{2}\right| & \leq C_{\lambda}|\beta|^{2}\|f\|_{L^{2}(2)}^{2}+\lambda\left\||\xi|^{2}\left(f-\bar{\alpha} e^{-\tau} \Delta f\right)\right\|_{L^{2}}^{2} \\
& \leq C_{\lambda}|\beta|^{2}\|f\|_{L^{2}}^{2}+\left(\lambda+C_{\lambda}|\beta|^{2}\right)\left\||\xi|^{2} f\right\|_{L^{2}}^{2}+\lambda \bar{\alpha}^{2} e^{-2 \tau}\left\||\xi|^{2} \Delta f\right\|_{L^{2}}^{2}
\end{aligned}
$$

Finally, integrating by parts the term $M_{3}$ and remarking that, for all $\mu>0$, there exists $C_{\mu}>0$ such that

$$
|\xi|^{3} \leq \mu|\xi|^{4}+C_{\mu}
$$

we can write

$$
\left|M_{3}\right| \leq\left\|K_{f}\right\|_{L^{\infty}}\left(\mu\left\||\xi|^{2}\left(f-\bar{\alpha} e^{-\tau} \Delta f\right)\right\|_{L^{2}}^{2}+C_{\mu}\left\|f-\bar{\alpha} e^{-\tau} \Delta f\right\|_{L^{2}}^{2}\right)
$$

Then, applying Lemma 2.2 part (a), we have

$$
\begin{aligned}
\left|M_{3}\right| \leq \mu\|f\|_{H^{1}}^{\frac{1}{2}}\|f\|_{L^{2}(2)}^{\frac{1}{2}}\left\||\xi|^{2}\left(f-\bar{\alpha} e^{-\tau} \Delta f\right)\right\|_{L^{2}}^{2} \\
+C_{\mu}\|f\|_{H^{1}}^{\frac{1}{2}}\|f\|_{L^{2}(2)}^{\frac{1}{2}}\left\|f-\bar{\alpha} e^{-\tau} \Delta f\right\|_{L^{2}}^{2} \\
\left.\leq \mu \sqrt{\delta}\left(\left\|\left.\xi\right|^{2} f\right\|_{L^{2}}^{2}+\bar{\alpha}^{2} e^{-2 \tau} \||\xi|^{2} \Delta f\right) \|_{L^{2}}^{2}\right) \\
+C_{\mu} \sqrt{\delta}\left(\|f\|_{L^{2}}^{2}+\bar{\alpha}^{2} e^{-2 \tau}\|\Delta f\|_{L^{2}}^{2}\right)
\end{aligned}
$$

since $f$ satisfies the bound (4.1).

Taking into account the estimates (4.44) and (4.46), using the fact that $|\beta|^{2} \leq \delta$ and applying the Cauchy-Schwarz inequality yield that, for all $\mu_{4}>0$, there exists a constant $C_{\mu_{4}}>0$ such that

$$
\begin{aligned}
& \partial_{\tau} E_{4}(\tau)+\left(\frac{1}{2}-\mu_{4}-C_{\mu_{4}}\left(\mu_{4} \sqrt{\delta}+\delta\right)\right)\left\||\xi|^{2} f\right\|_{L^{2}}^{2} \\
& +\left(1+\bar{\alpha} e^{-\tau}\right)\left\||\xi|^{2} \nabla f\right\|_{L^{2}}^{2} \\
& +\bar{\alpha} e^{-\tau}\left(1+\frac{\bar{\alpha}}{2} e^{-\tau}-\bar{\alpha} e^{-\tau}\left(\mu_{4} \sqrt{\delta}+\mu_{4}\right)\right)\left\||\xi|^{2} \Delta f\right\|_{L^{2}}^{2} \\
& +\bar{\epsilon} e^{-2 \tau}\left(\left\||\xi|^{2} \Delta f\right\|_{L^{2}}^{2}+32\|f\|_{L^{2}}^{2}+\bar{\alpha} e^{-\tau}\left\||\xi|^{2} \nabla \Delta f\right\|_{L^{2}}^{2}\right) \\
& -\left(8+8 \bar{\alpha} e^{-\tau}\right)\||\xi| f\|_{L^{2}}^{2}-\bar{\epsilon} e^{-2 \tau}\left(24\||\xi| \nabla f\|_{L^{2}}^{2}+8 \bar{\alpha} e^{-\tau}\||\xi| \Delta f\|_{L^{2}}^{2}\right) \\
& \leq C_{\mu_{4}} \frac{|\beta|^{2}}{\nu^{2}}\left(\bar{\epsilon}^{2}+\bar{\alpha}^{2}\right) e^{-2 \tau}+C_{\mu_{4}}\left(|\beta|^{2}+\sqrt{\delta}\right)\|f\|_{L^{2}}^{2} \\
& +\bar{\alpha}^{2} e^{-2 \tau} C_{\mu_{4}} \sqrt{\delta}\|\Delta f\|_{L^{2}}^{2}
\end{aligned}
$$

For any $\lambda>0$, we can write

$$
\||\xi| f\|_{L^{2}}^{2} \leq \frac{\lambda}{2}\left\||\xi|^{2} f\right\|_{L^{2}}^{2}+\frac{1}{2 \lambda}\|f\|_{L^{2}}^{2}
$$


Likewise, for any $\eta>0$, there exists a constant $C_{\eta}>0$ such that

$$
\begin{aligned}
& \bar{\epsilon} e^{-2 \tau}\||\xi| \nabla f\|_{L^{2}}^{2} \leq \bar{\epsilon} e^{-2 \tau}\left(\eta\left\||\xi|^{2} \nabla f\right\|_{L^{2}}^{2}+C_{\eta}\|\nabla f\|_{L^{2}}^{2}\right) \\
& \bar{\epsilon} \bar{\alpha} e^{-3 \tau}\||\xi| \Delta f\|_{L^{2}}^{2} \leq \bar{\epsilon} \bar{\alpha} e^{-3 \tau}\left(\eta\left\||\xi|^{2} \Delta f\right\|_{L^{2}}^{2}+C_{\eta}\|\Delta f\|_{L^{2}}^{2}\right)
\end{aligned}
$$

Thus, for any $\mu>0$ and any $\lambda>0$, there exists a constant $C_{\mu}>0$ such that Inequality (4.46) becomes

$$
\begin{aligned}
& \partial_{\tau} E_{4}(\tau)+\left(\frac{1}{2}-\mu-C_{\mu}(\mu \sqrt{\delta}+\delta)-4 \lambda\left(1+\bar{\alpha} e^{-\tau}\right)\right)\left\||\xi|^{2} f\right\|_{L^{2}}^{2} \\
& +\left(1+\bar{\alpha} e^{-\tau}-\bar{\epsilon} e^{-2 \tau} C_{\mu}\right)\left\||\xi|^{2} \nabla f\right\|_{L^{2}}^{2} \\
& +\bar{\alpha} e^{-\tau}\left(1+\frac{\bar{\alpha}}{2} e^{-\tau}-\bar{\alpha} e^{-\tau}\left(\mu \sqrt{\delta}+\mu+C_{\mu} \bar{\epsilon} e^{-2 \tau}\right)\right)\left\||\xi|^{2} \Delta f\right\|_{L^{2}}^{2} \\
& +\bar{\epsilon} e^{-2 \tau}\left(\left\||\xi|^{2} \Delta f\right\|_{L^{2}}^{2}+32\|f\|_{L^{2}}^{2}+\bar{\alpha} e^{-\tau}\left\||\xi|^{2} \nabla \Delta f\right\|_{L^{2}}^{2}\right) \\
& \leq C_{\mu} \frac{|\beta|^{2}}{\nu^{2}}\left(\bar{\epsilon}^{2}+\bar{\alpha}^{2}\right) e^{-2 \tau}+\left(\frac{4}{\lambda}(1+\bar{\alpha})+C_{\mu}\left(|\beta|^{2}+\sqrt{\delta}\right)\|f\|_{L^{2}}^{2}\right. \\
& +C_{\mu} \bar{\epsilon} e^{-2 \tau}\|\nabla f\|_{L^{2}}^{2}+\left(C_{\mu} \bar{\epsilon} \bar{\alpha} e^{-3 \tau}+\bar{\alpha}^{2} e^{-2 \tau} C_{\mu} \sqrt{\delta}\right)\|\Delta f\|_{L^{2}}^{2}
\end{aligned}
$$

We finally remark that

$$
\begin{aligned}
\left\||\xi|^{2}\left(f-\bar{\alpha} e^{-\tau} \Delta f\right)\right\|_{L^{2}}^{2}= & \left\||\xi|^{2} f\right\|_{L^{2}}^{2}+2 \bar{\alpha} e^{-\tau}\left\||\xi|^{2} \nabla f\right\|_{L^{2}}^{2} \\
& +\bar{\alpha}^{2} e^{-2 \tau}\left\||\xi|^{2} \Delta f\right\|_{L^{2}}^{2}-16 \bar{\alpha} e^{-\tau}\||\xi| f\|_{L^{2}}^{2},
\end{aligned}
$$

and thus that

$$
\begin{gathered}
\left\||\xi|^{2}\left(f-\bar{\alpha} e^{-\tau} \Delta f\right)\right\|_{L^{2}}^{2} \leq\left\||\xi|^{2} f\right\|_{L^{2}}^{2}+2 \bar{\alpha} e^{-\tau}\left\||\xi|^{2} \nabla f\right\|_{L^{2}}^{2} \\
+\bar{\alpha}^{2} e^{-2 \tau}\left\||\xi|^{2} \Delta f\right\|_{L^{2}}^{2}
\end{gathered}
$$

Next, we set $\mu=4 \lambda=\frac{1}{12}$, for example. Thus, (4.47), together with (4.48), implies that

$$
\begin{aligned}
& \partial_{\tau} E_{4}(\tau)+\frac{1}{2} E_{4}(\tau)+\left(\frac{1}{12}-C_{4}\left(\sqrt{\delta}+\bar{\alpha} e^{-\tau}+\delta\right)\right)\left\||\xi|^{2} f\right\|_{L^{2}}^{2} \\
& +\left(1+\frac{\bar{\alpha}}{2} e^{-\tau}-\bar{\epsilon} e^{-2 \tau} C_{4}\right)\left\||\xi|^{2} \nabla f\right\|_{L^{2}}^{2} \\
& +\bar{\alpha} e^{-\tau}\left(1+\frac{\bar{\alpha}}{4} e^{-\tau}-\bar{\alpha} e^{-\tau}\left(\sqrt{\delta}+\frac{1}{12}+C_{4} \bar{\epsilon} e^{-2 \tau}\right)\right)\left\||\xi|^{2} \Delta f\right\|_{L^{2}}^{2} \\
& +\bar{\epsilon} e^{-2 \tau}\left(\left\||\xi|^{2} \Delta f\right\|_{L^{2}}^{2}+32\|f\|_{L^{2}}^{2}+\bar{\alpha} e^{-\tau}\left\||\xi|^{2} \nabla \Delta f\right\|_{L^{2}}^{2}\right) \\
& \leq C_{4} \delta\left(\bar{\epsilon}^{2}+\bar{\alpha}^{2}\right) e^{-2 \tau}+\left(48+C_{4}\left(\bar{\alpha} e^{-\tau}+\sqrt{\delta}\right)\|f\|_{L^{2}}^{2}\right. \\
& +C_{4} \bar{\epsilon} e^{-2 \tau}\|\nabla f\|_{L^{2}}^{2}+\left(C_{4} \bar{\epsilon} \bar{\alpha} e^{-3 \tau}+\bar{\alpha}^{2} e^{-2 \tau} C_{4} \sqrt{\delta}\right)\|\Delta f\|_{L^{2}}^{2}
\end{aligned}
$$

The lemma 4.4 is thus proved. 
We have obtained energy estimates of the functionals $E_{3}$ and $E_{4}$. In order to obtain an energy estimate of $f$ in the space $H^{2}(2)$, we introduce a final functional, which is a linear combination of $E_{3}$ and $E_{4}$. In order to choose appropriate coefficients, we begin by remarking that

$$
3\|f\|_{L^{2}}^{2} \leq 2\|\nabla F\|_{L^{2}}^{2}+\|\nabla f\|_{L^{2}}^{2} .
$$

Thus, choosing $b>12 \times 16=192$, we introduce the functional

$$
E_{5}(\tau)=b E_{3}(\tau)+E_{4}(\tau)
$$

Then, we have the following lemma.

LEMMA 4.5. Let $f \in L^{\infty}\left(\left[\tau_{0}, \tau_{1}\right], H^{2}(2)\right)$ be a solution of (3.14) satisfying the bound (4.1). There exist three constants $K_{0}>0, K_{1}>0$ and $K_{2}>0$ such that

$$
\|f\|_{H^{1}}^{2}+\bar{\alpha} e^{-\tau}\|\Delta f\|_{L^{2}}^{2}+\left\|\left.\xi\right|^{2}\left(f-\bar{\alpha} e^{-\tau} \Delta f\right)\right\|_{L^{2}}^{2} \leq K_{1} E_{5}(\tau)
$$

$$
E_{5}(\tau) \leq K_{2}\left(\|f\|_{H^{1}}^{2}+\bar{\alpha} e^{-\tau}\|\Delta f\|_{L^{2}}^{2}+\left\||\xi|^{2} f\right\|_{L^{2}}^{2}+\bar{\alpha}^{2} e^{-2 \tau}\left\||\xi|^{2} \Delta f\right\|_{L^{2}}^{2}\right)
$$

Moreover, $E_{5}(\tau)$ satisfies the following inequality for all $\tau \in\left[\tau_{0}, \tau_{1}\right]$,

$$
E_{5}(\tau) \leq e^{-\theta\left(\tau-\tau_{0}\right)} E_{5}\left(\tau_{0}\right)+\frac{K_{0} \delta}{2-\theta}\left(\bar{\alpha}^{2}+\bar{\epsilon}\right) e^{-\theta \tau}
$$

with $0<\theta<\frac{1}{2}$.

Proof : Using the definition of $E_{5}$, it is easy to see that Inequality (4.51) is verified. The estimate (4.52) is a direct consequence of the definition (4.50) of $E_{5}$ and the lemmas 3.4 and 2.4 .

It remains to prove Inequality (4.53). Using (4.34), (4.49) and choosing the constants $\bar{\epsilon}, \delta$ and $\bar{\alpha}$ sufficiently small, we show that there exists a constant $K_{0}>0$ such that $E_{5}$ satisfies the following inequality

$$
\begin{aligned}
& \partial_{\tau} E_{5}(\tau)+\theta E_{5}(\tau)+\bar{\epsilon} e^{-2 \tau}\left(b\left(\frac{15}{4}+\frac{5 \theta}{2}\right)\|\Delta F\|_{L^{2}}^{2}+\frac{5 b}{2}\|\Delta f\|_{L^{2}}^{2}+\frac{b}{4}\|\nabla \Delta f\|_{L^{2}}^{2}\right) \\
& +\bar{\epsilon} e^{-2 \tau}\left(\left\||\xi|^{2} \Delta f\right\|_{L^{2}}^{2}+32\|f\|_{L^{2}}^{2}+\bar{\alpha} e^{-\tau}\left\||\xi|^{2} \nabla \Delta f\right\|_{L^{2}}^{2}\right) \\
& \leq K_{0} \delta\left(\bar{\alpha}^{2}+\bar{\epsilon}\right) e^{-2 \tau}
\end{aligned}
$$

where $0<\theta<\frac{1}{2}$.

Integrating the above inequality between $\tau_{0}$ and $\tau>\tau_{0}$ and applying the Gronwall inequality, we obtain

$$
\begin{aligned}
E_{5}(\tau) & \leq e^{-\theta\left(\tau-\tau_{0}\right)} E_{5}\left(\tau_{0}\right)+\frac{K_{0} \delta}{2-\theta}\left(\bar{\alpha}^{2}+\bar{\epsilon}\right) e^{-\theta \tau}\left(e^{-\tau_{0}(2-\theta)}-e^{-\tau(2-\theta)}\right) \\
& \leq e^{-\theta\left(\tau-\tau_{0}\right)} E_{5}\left(\tau_{0}\right)+\frac{K_{0} \delta}{2-\theta}\left(\bar{\alpha}^{2}+\bar{\epsilon}\right) e^{-\theta \tau}
\end{aligned}
$$

with $0<\theta<\frac{1}{2}$.

\section{Proof of Theorem 3.3 and convergence when $\epsilon$ tends to zero}

In this section, we first prove Theorem 3.3. Next, passing to the limit, when $\epsilon$ tends to zero, we prove Theorem 1.1 and Corollary 1.2. 
5.1. Proof of Theorem 3.3. Let $\gamma$ be a small positive constant and let $W_{0}$ belong to $H^{2}(2)$. Theorem 2.6 implies that there exists a solution $W \in$ $C\left(\left[\tau_{0}, \hat{\tau}\right], H^{2}(2)\right)$ of Equation $(3.3)$ with $W(0)=W_{0}$, where $\hat{\tau}=\log \left(T+t_{\max }\right)$.

We recall that $W_{\epsilon}(\xi, \tau)=\beta G(\xi)+f_{\epsilon}(\xi, \tau)$.

Thus, the global existence of $W_{\epsilon}$ is a direct consequence of the global existence of $f_{\epsilon}$.

We also know that the solution $f_{\epsilon}$ of Equation (3.14) exists on $\left[\tau_{0}, \hat{\tau}\right]$ and belongs to $C\left(\left[\tau_{0}, \hat{\tau}\right], H^{2}(2)\right)$.

We suppose that $W_{0}$ satisfies the bound (3.11).

Using the continuous injection of $L^{2}(2)$ into $L^{1}\left(\mathbb{R}^{2}\right)$, we can write that

$$
|\beta| \leq C \gamma_{0}
$$

where $\beta$ is given by (3.13) and $C>0$ is a constant.

Therefore, we have the following bound

$$
\begin{aligned}
|\beta|^{2}+\left\|f_{\epsilon}\left(\tau_{0}\right)\right\|_{H^{1}}^{2}+\bar{\alpha}\left\|\Delta f_{\epsilon}\left(\tau_{0}\right)\right\|_{L^{2}}^{2} & \\
& +\left\||\xi|^{2} f_{\epsilon}\left(\tau_{0}\right)\right\|_{L^{2}}^{2}+\bar{\alpha}\left\||\xi|^{2} \Delta f_{\epsilon}\left(\tau_{0}\right)\right\|_{L^{2}}^{2} \leq \tilde{C} \gamma_{0},
\end{aligned}
$$

where $\tilde{C}>0$ is a constant.

Let $\delta=\frac{\tilde{C} \gamma_{0}}{\kappa}$, where $0<\kappa<\frac{1}{4}$ is a constant that will be made more precise later. By continuity of the solutions, there exists a time $\tau_{1}$, with $\tau_{0}<\tau_{1} \leq \hat{\tau}$, such that, for all $\tau \leq \tau_{1}, f_{\epsilon}$ satisfies the bound (4.1). Then, according to (4.53), we have, for $\tau_{0} \leq \tau \leq \tau_{1}$

$$
E_{5}(\tau) \leq e^{-\theta\left(\tau-\tau_{0}\right)} E_{5}(0)+\frac{K_{0} \delta}{2-\theta}\left(\bar{\alpha}^{2}+\bar{\epsilon}\right) e^{-\theta \tau}
$$

where $0<\theta<\frac{1}{2}$.

Let

$$
A(\tau)=\left\|f_{\epsilon}(\tau)\right\|_{H^{1}}^{2}+\left\||\xi|^{2} f_{\epsilon}(\tau)\right\|_{L^{2}}^{2} .
$$

In order to complete the proof of the global existence of $f_{\epsilon}$, it remains to show that $A(\tau)<\delta$, for all $\tau, \tau_{0} \leq \tau \leq \tau_{1}$.

We recall that

$$
\begin{aligned}
\left\||\xi|^{2}\left(f_{\epsilon}-\bar{\alpha} e^{-\tau} \Delta f_{\epsilon}\right)\right\|_{L^{2}}^{2} & =\left\||\xi|^{2} f_{\epsilon}\right\|_{L^{2}}^{2}-16 \bar{\alpha} e^{-\tau}\left\||\xi| f_{\epsilon}\right\|_{L^{2}}^{2} \\
& +2 \bar{\alpha} e^{-\tau}\left\||\xi|^{2} \nabla f_{\epsilon}\right\|_{L^{2}}^{2}+\bar{\alpha}^{2} e^{-2 \tau}\left\||\xi|^{2} \Delta f_{\epsilon}\right\|_{L^{2}}^{2}
\end{aligned}
$$

Thus,

$$
\left\||\xi|^{2} f_{\epsilon}\right\|_{L^{2}}^{2} \leq\left\||\xi|^{2}\left(f_{\epsilon}-\bar{\alpha} e^{-\tau} \Delta f_{\epsilon}\right)\right\|_{L^{2}}^{2}+16 \bar{\alpha} e^{-\tau}\left\||\xi| f_{\epsilon}\right\|_{L^{2}}^{2}
$$

We also have, for all $\mu>0$,

$$
\left\||\xi| f_{\epsilon}\right\|_{L^{2}}^{2} \leq \frac{\mu}{2}\left\||\xi|^{2} f_{\epsilon}\right\|_{L^{2}}^{2}+\frac{1}{2 \mu}\left\|f_{\epsilon}\right\|_{L^{2}}^{2}
$$

Choosing $\mu=\frac{1}{16}$, we can write

$$
\frac{1}{2}\left\||\xi|^{2} f_{\epsilon}\right\|_{L^{2}}^{2} \leq\left\||\xi|^{2}\left(f_{\epsilon}-\bar{\alpha} e^{-\tau} \Delta f_{\epsilon}\right)\right\|_{L^{2}}^{2}+128 \bar{\alpha} e^{-\tau}\left\|f_{\epsilon}\right\|_{L^{2}}^{2}
$$

Therefore, using Inequality (4.51) of Lemma 4.5, we obtain that there exists a constant $\tilde{K}_{1}>K_{1}$ such that

$$
A(\tau) \leq \tilde{K}_{1} E_{5}(\tau)
$$


On the other hand, we have, by Inequality (4.52) of Lemma 4.5,

$$
E_{5}(\tau) \leq K_{2}\left(A(\tau)+\bar{\alpha} e^{-\tau}\left\|\Delta f_{\epsilon}(\tau)\right\|_{L^{2}}^{2}+\bar{\alpha}^{2} e^{-2 \tau}\left\||\xi|^{2} \Delta f_{\epsilon}(\tau)\right\|_{L^{2}}^{2}\right)
$$

Now, let

$$
K=\tilde{K}_{1} \max \left(K_{0}, K_{2}\right)
$$

and choose

$$
\kappa=\frac{1}{4} \min (1, K)
$$

where $K_{0}$ is the constant given in Inequality (4.53). Suppose that $T$ is large enough (which is equivalent to saying that $\bar{\epsilon}$ and $\bar{\alpha}$ are small enough) such that

$$
K\left(\bar{\alpha}^{2}+\bar{\epsilon}\right) \leq \frac{1}{8}, \text { and } \bar{\alpha} \leq \frac{1}{4} .
$$

Then, using Estimate (4.53) and Inequalities (5.2) (5.3), we obtain

$$
\begin{aligned}
A(\tau) & \leq K_{1} E_{5}(\tau) \\
& \leq K_{1} e^{-\theta\left(\tau-\tau_{0}\right)} E_{5}\left(\tau_{0}\right)+K_{1} K_{0} \frac{\delta}{2-\theta}\left(\bar{\alpha}^{2}+\bar{\epsilon}\right) e^{-\theta \tau} \\
& \leq K e^{-\theta\left(\tau-\tau_{0}\right)}\left(A\left(\tau_{0}\right)+\bar{\alpha}\left\|\Delta f_{\epsilon}\left(\tau_{0}\right)\right\|_{L^{2}}^{2}+\bar{\alpha}^{2}\left\||\xi|^{2} \Delta f_{\epsilon}\left(\tau_{0}\right)\right\|_{L^{2}}^{2}\right)+\frac{\delta}{4} e^{-\theta \tau} \\
& <\delta
\end{aligned}
$$

Therefore, $A(\tau)$ remains small on $\left[\tau_{0}, \tau_{1}\right]$. We conclude that $\tau_{1}=\hat{\tau}$. On the other hand, Inequalities (4.51) and (4.53) imply that the solution $f_{\epsilon}$ of Equation (3.14) satisfies the following bound $\forall \tau_{0} \leq \tau \leq \hat{\tau}$,

$$
\begin{aligned}
\left\|f_{\epsilon}(\tau)\right\|_{H^{1}}^{2}+\bar{\alpha} e^{-\tau} & \left\|\Delta f_{\epsilon}(\tau)\right\|_{L^{2}}^{2}+\left\||\xi|^{2}\left(f_{\epsilon}(\tau)-\bar{\alpha} e^{-\tau} \Delta f_{\epsilon}\right)\right\|_{L^{2}}^{2} \\
& \leq K_{1} e^{-\theta\left(\tau-\tau_{0}\right)} E_{5}\left(\tau_{0}\right)+\frac{K_{1} K_{0} \delta}{2-\theta}\left(\bar{\alpha}^{2}+\bar{\epsilon}\right) e^{-\theta \tau}
\end{aligned}
$$

where $0<\theta<\frac{1}{2}$. Therefore,

$$
\left\|f_{\epsilon}(\tau)-\bar{\alpha} e^{-\tau} \Delta f_{\epsilon}\right\|_{L^{2}(2)}^{2} \leq C e^{-\theta \tau},
$$

where $C$ is a positive constant.

The above inequality implies that the $H^{2}(2)$-norm of $f_{\epsilon}$ remains bounded for all $\tau \in\left[\tau_{0}, \hat{\tau}\right]$. Thus, $\hat{\tau}=+\infty$ and $f_{\epsilon}$ belongs to the space $L^{\infty}\left(\left[\tau_{0},+\infty\left[, H^{2}(2)\right)\right.\right.$.

5.2. Convergence when $\epsilon$ tends to zero. Before we begin the proof of Theorem 1.1, we give the following lemmas, which will be useful later.

Lemma 5.1. Let $w \in L^{2}(2)$ and let $z=(I-\alpha \Delta)^{-1} w$. Then, there exists a positive constant $C$ such that we have the following inequalities:
a) $\|z\|_{L^{2}}^{2}+2 \alpha\|\nabla z\|_{L^{2}}^{2}+\alpha^{2}\|\Delta z\|_{L^{2}}^{2} \leq C\|w\|_{L^{2}}^{2}$.
b) $\left\||x|^{2} z\right\|_{L^{2}}^{2}+2 \alpha\left\||x|^{2} \nabla z\right\|_{L^{2}}^{2}+\alpha^{2}\left\||x|^{2} \Delta z\right\|_{L^{2}}^{2} \leq C\|w\|_{L^{2}(2)}^{2}$. 
Proof : We have

$$
z-\alpha \Delta z=w .
$$

Thus, in order to prove Inequalities a) and b), it is sufficient to take the scalar product of (5.8) with $z-\alpha \Delta z$ and $|x|^{4}(z-\alpha \Delta z)$ respectively.

LEMMA 5.2. 1) Let $f$ and $g$ be two functions such that $g \in L^{2}(1) \cap H^{1}\left(\mathbb{R}^{2}\right)$, $\nabla f \in L^{4}\left(\mathbb{R}^{2}\right) \cap L^{\infty}\left(\mathbb{R}^{2}\right)$ and $\Delta f \in L^{2}\left(\mathbb{R}^{2}\right) \cap L^{4}\left(\mathbb{R}^{2}\right)$. Also let

$h=(I-\alpha \Delta)^{-1}(f .(I-\alpha \Delta) g)-f g$. Then, there exists a positive constant $C$ such that we have the following inequalities:

a) $\|h\|_{L^{2}}^{2}+\alpha\|\nabla h\|_{L^{2}}^{2} \leq C_{\alpha}\left(\|\Delta f\|_{L^{2}}^{2}\|g\|_{H^{1}}^{2}+\|\nabla f\|_{L^{4}}^{2}\|\nabla g\|_{L^{2}}^{2}\right)$.

b) $\||x| h\|_{L^{2}}^{2}+\alpha\||x| \nabla h\|_{L^{2}}^{2} \leq C_{\alpha}\left(\|h\|_{L^{2}}^{2}+\|\Delta f\|_{L^{4}}^{2}\||x| g\|_{L^{2}}^{2}\right.$

$$
\left.+\|\nabla f\|_{L^{\infty}}^{2}\left(\|g\|_{L^{2}}^{2}+\||x| g\|_{L^{2}}^{2}\right)\right),
$$

where $C_{\alpha}=C\left(\alpha+\alpha^{2}\right)$.

2) Let $\bar{f}$ and $\bar{g}$ be two functions such that $\bar{f} \in L^{\infty}\left(\mathbb{R}^{2}\right), \nabla \bar{g} \in L^{2}(2)$ and $\Delta \bar{g} \in L^{2}(2)$. Also let $k=(I-\alpha \Delta)^{-1}(\bar{f} . \nabla(I-\alpha \Delta) \bar{g})$. Then, we have the following inequalities:

$$
\begin{aligned}
& \text { c) }\|k\|_{L^{2}}^{2}+\alpha\|\nabla k\|_{L^{2}}^{2} \leq C\|\bar{f}\|_{L^{\infty}}^{2}\left(\|\nabla \bar{g}\|_{L^{2}}^{2}+\alpha\|\Delta \bar{g}\|_{L^{2}}^{2}\right) \text {. } \\
& \text { d) }\left\||x|^{2} k\right\|_{L^{2}}^{2}+\alpha\left\||x|^{2} \nabla k\right\|_{L^{2}}^{2} \leq C_{\alpha}\left(\|k\|_{L^{2}}^{2}\right. \\
& \left.+\|\bar{f}\|_{L^{\infty}}^{2}\left(\left\||x|^{2} \nabla \bar{g}\right\|_{L^{2}}^{2}+\left\||x|^{2} \Delta \bar{g}\right\|_{L^{2}}^{2}\right)\right) .
\end{aligned}
$$

Proof : Using the expression of $h$, we can write

$$
h-\alpha \Delta h=\alpha \Delta f g+2 \alpha \nabla f . \nabla g
$$

Taking the scalar product of Equation (5.9) with $h$ and integrating by parts, we can write the following inequality

$$
\|h\|_{L^{2}}^{2}+\alpha\|\nabla h\|_{L^{2}}^{2} \leq C \alpha\|h\|_{L^{4}}\left(\|\Delta f\|_{L^{2}}\|g\|_{L^{4}}+\|\nabla f\|_{L^{4}}\|\nabla g\|_{L^{2}}\right)
$$

Then, applying the Cauchy-Schwarz inequality, we obtain Inequality a).

In order to obtain Inequality b), it is sufficient to take the scalar product of Equation (5.9) with $|x|^{2} h$.

Inequalities c) and d) can be shown by the same way.

Now, we prove the Theorem 1.1. In the previous section, we have showed that the solution $f_{\epsilon}$ of Equation (3.14) belongs to the space $C^{0}\left(\left[\tau_{0},+\infty\right), H^{2}(2)\right)$ and that Estimate (5.6) is satisfied, $\forall \tau \geq \tau_{0}$. In particular,

$\left\|f_{\epsilon}(\tau)-\bar{\alpha} e^{-\tau} \Delta f_{\epsilon}(\tau)\right\|_{L^{2}(2)}$ is bounded uniformly in $\epsilon$ and decays exponentially to 0 .

On the other hand, one can show, according to Equation (3.14), that $\partial_{\tau}\left(f_{\epsilon}-\bar{\alpha} e^{-\tau} \Delta f_{\epsilon}\right)$ is uniformly bounded in $\epsilon$ in the space $L^{\infty}\left(\left[\tau_{0}, \tau\right], H^{-2}\left(\mathbb{R}^{2}\right)\right)$. Therefore,

$$
\partial_{\tau} f_{\epsilon} \in L^{\infty}\left(\left[\tau_{0}, \tau\right], L^{2}\left(\mathbb{R}^{2}\right)\right)
$$

Let $O$ be a bounded open set in $\mathbb{R}^{2}$. Property (5.11) implies that the family $\left(f_{\epsilon}\right)$, $\epsilon>0$, is equicontinuous in $C^{0}\left(\left[\tau_{0}, \tau\right], L^{2}(O)\right)$.

Ascoli's theorem allows us to extract a subsequence still denoted by $f_{\epsilon}$ such that 
$f_{\epsilon}$ converges strongly to $f$ in $C^{0}\left(\left[\tau_{0}, \tau\right], H^{-1}(O)\right)$. Next, the interpolation between $H^{-1}(O)$ and $H^{2}(O)$ allows us to deduce that

$$
f_{\epsilon} \longrightarrow f \text { strongly in } C^{0}\left(\left[\tau_{0}, \tau\right], H^{s}(O)\right), \forall s<2 .
$$

Let us note that, by the Hölder inequality, we have

$$
\left\||\xi|^{\frac{3}{2}} f_{\epsilon}\right\|_{L^{2}} \leq\left\|f_{\epsilon}\right\|_{L^{2}}^{\frac{1}{4}}\left\||\xi|^{2} f_{\epsilon}\right\|_{L^{2}}^{\frac{3}{4}}
$$

Then, using property (5.12) and the fact that $f_{\epsilon}$ is uniformly bounded in

$$
L^{\infty}\left(\left[\tau_{0},+\infty\right], L^{2}(2)\right)
$$

, we deduce that, for any $\tau>0$,

$$
|\xi|^{\frac{3}{2}} f_{\epsilon} \longrightarrow|\xi|^{\frac{3}{2}} f \text { strongly in } C^{0}\left([0, \tau], L^{2}(O)\right) .
$$

Using Lemma 2.1 part b), together with the properties (5.12) and (5.14), we deduce that

$$
K_{f_{\epsilon}} \longrightarrow K_{f} \text { strongly in } L^{\infty}\left(\left[\tau_{0}, \tau\right], L^{\infty}(O)\right) .
$$

Also, using Lemma 2.1 part b) and Property (5.12), we deduce that

$$
\nabla K_{f_{\epsilon}} \longrightarrow \nabla K_{f} \text { strongly in } L^{\infty}\left(\left[\tau_{0}, \tau\right], L^{4}(O)\right) .
$$

These convergences allow us to show that $f$ satisfies the equation (3.14) in the weak sense for $\epsilon=0$. To this end, we take the $L^{2}$-product of (3.14) with any function $\phi \in C_{0}^{\infty}\left(\mathbb{R}^{+} \times \mathbb{R}^{2}, \mathbb{R}^{2}\right)$ such that $\operatorname{supp}(\phi) \subset\left[\tau_{0}, \tau\right] \times O$.

We will only prove the convergence of the nonlinear term

$\int_{\tau_{0}}^{\tau} \int_{O} K_{f_{\epsilon}} \cdot \nabla\left(f_{\epsilon}-\bar{\alpha} e^{-\tau} \Delta f_{\epsilon}\right) \cdot \phi d \xi d \tau$ since, for the other terms, the convergence is easier to prove.

Let $\phi \in C_{0}^{\infty}\left(\mathbb{R}^{+} \times \mathbb{R}^{2}, \mathbb{R}^{2}\right)$ such that $\operatorname{supp}(\phi) \subset\left[\tau_{0}, \tau\right] \times O$.

Then, integrating by parts, we obtain

$$
\begin{aligned}
& \int_{\tau_{0}}^{\tau} \int_{O} K_{f_{\epsilon}} \cdot \nabla\left(f_{\epsilon}-\bar{\alpha} e^{-\tau} \Delta f_{\epsilon}\right) \phi d \xi d \tau=\int_{0}^{\tau} \int_{O} K_{f_{\epsilon}} \cdot \nabla \phi\left(f_{\epsilon}-\bar{\alpha} e^{-\tau} \Delta f_{\epsilon}\right) d \xi d \tau \\
& =\int_{\tau_{0}}^{\tau} \int_{O} K_{f_{\epsilon}} \cdot \nabla \phi f_{\epsilon} \\
& +\bar{\alpha} e^{-\tau} \int_{0}^{\tau} \int_{O} \sum_{k=1}^{2}\left(\partial_{k} K_{f_{\epsilon}} \cdot \nabla \phi \partial_{k} f_{\epsilon}+K_{f_{\epsilon}} \cdot \nabla \partial_{k} \phi \partial_{k} f_{\epsilon}\right) d \xi d \tau
\end{aligned}
$$

Using Properties (5.15), (5.16) and (5.12), we deduce that

$$
\int_{\tau_{0}}^{\tau} \int_{O} K_{f_{\epsilon}} \cdot \nabla \phi\left(f_{\epsilon}-\bar{\alpha} e^{-\tau} \Delta f_{\epsilon}\right) d \xi d \tau \rightarrow \int_{\tau_{0}}^{\tau} \int_{O} K_{f} \cdot \nabla \phi\left(f-\bar{\alpha} e^{-\tau} \Delta f\right) d \xi d \tau .
$$

Therefore, $f$ satisfies the equation (3.14) in the weak sense for $\epsilon=0$.

On the other hand, remarking that the estimates proved in the Section 4 are uniform with respect to $\epsilon$, we can then obtain the same estimates for the limiting solution $f$ with $\epsilon=0$. We thus have shown that $f$ satisfies the same decay rate in time as $f_{\epsilon}$ and we have

$$
\|f(\tau)\|_{H^{1}}^{2}+\bar{\alpha}\|\Delta f(\tau)\|_{L^{2}}^{2}+\left\||\xi|^{2}\left(f(\tau)-\bar{\alpha} e^{-\tau} \Delta f\right)\right\|_{L^{2}(2)}^{2} \leq \delta e^{-\theta \tau}
$$


where $0<\theta<\frac{1}{2}$ and $C>0$ is a constant.

Now, we prove the uniqueness of solutions of Equation (1.6). For this purpose, we return to Equation (1.1) written in the original variables $(x, t)$.

Suppose that $w_{1}$ and $w_{2}$ are two solutions of Equation (1.1) such that $w_{1}(0, x)=$ $w_{2}(0, x)=w_{0}(x)$ and let $u_{1}$ and $u_{2}$ be the velocity fields associated to the vorticities $w_{1}$ and $w_{2}$ respectively.

Then, $w=w_{1}-w_{2}$ satisfies the following equation

$$
\begin{aligned}
& \partial_{\tau}(w-\alpha \Delta w)-\nu \Delta w+u_{1} \cdot \nabla(w-\alpha \Delta w)+\left(u_{1}-u_{2}\right) \cdot \nabla\left(w_{2}-\alpha \Delta w_{2}\right)=0 \\
& w(0, x)=0
\end{aligned}
$$

According to Lemma 2.3, we have that

$$
\left\|u_{1}-u_{2}\right\|_{L^{2}} \leq C\||x| w\|_{L^{2}} .
$$

This indicated that the uniqueness of solutions should be proved by using a weighted norm.

Before we start the proof of the uniqueness of solutions, we will give the following bounds, which will be useful later.

Using the Fourier transform of $u$ and the continuous injection of $L^{2}(2)$ into $L^{1}\left(\mathbb{R}^{2}\right)$, we can write

$$
\begin{aligned}
\left\|\nabla u_{1}\right\|_{L^{\infty}} & \leq C\left\|\widehat{\nabla u_{1}}\right\|_{L^{1}} \leq C\left\|\widehat{w_{1}}\right\|_{L^{1}} \\
& \leq C\left\|\widehat{w_{1}}\right\|_{L^{2}(2)} \leq C\left(\left\|w_{1}\right\|_{L^{2}}+\left\|\Delta w_{1}\right\|_{L^{2}}\right) \leq C,
\end{aligned}
$$

where $C>0$ is a constant.

We also have by Lemma 2.1 part(d) that

$$
\begin{aligned}
\left\|\Delta u_{1}\right\|_{L^{4}} & \leq C\left\|\Delta u_{1}\right\|_{H^{1}} \leq C\left(\left\|\Delta u_{1}\right\|_{L^{2}}+\left\|\nabla \Delta u_{1}\right\|_{L^{2}}\right) \\
& \leq C\left(\left\|\nabla w_{1}\right\|_{L^{2}}+\left\|\Delta w_{1}\right\|_{L^{2}} \leq C .\right.
\end{aligned}
$$

As we have explained before, in order to prove that $w=0$, we will write an energy estimation of $w$ in the space $L^{2}(2)$. As a first step, we begin by performing an estimation of $\|w\|_{L^{2}}+\sqrt{\alpha}\|\nabla w\|_{L^{2}}$. For this purpose, we take the scalar product of Equation (5.18) with $w$. We obtain, after some integrations by parts,

$$
\begin{aligned}
& \frac{1}{2} \partial_{t}\left(\|w\|_{L^{2}}^{2}+\alpha\|\nabla w\|_{L^{2}}^{2}\right)+\nu\|\nabla w\|_{L^{2}}^{2} \\
& \quad \leq C\left\|\nabla u_{1}\right\|_{L^{\infty}}\|\nabla w\|_{L^{2}}^{2}+C\left\|u_{1}-u_{2}\right\|_{L^{\infty}}\left\|\Delta w_{2}\right\|_{L^{2}}\|\nabla w\|_{L^{2}}
\end{aligned}
$$

Therefore, using (5.19) and Lemma 2.2 part a), then applying the Cauchy-Schwarz inequality, we obtain

$$
\begin{aligned}
\frac{1}{2} \partial_{t}\left(\|w\|_{L^{2}}^{2}+\alpha \|\right. & \left.\nabla w \|_{L^{2}}^{2}\right)+\nu\|\nabla w\|_{L^{2}}^{2} \\
& \leq C\|\nabla w\|_{L^{2}}^{2}+C\|w\|_{L^{2}(2)}^{\frac{1}{2}}\|w\|_{H^{1}}^{\frac{1}{2}}\|\nabla w\|_{L^{2}} \\
& \leq C\|\nabla w\|_{L^{2}}^{2}+C\|w\|_{L^{2}(2)}\|w\|_{H^{1}}
\end{aligned}
$$

In order to obtain an estimation of $w$ in $L^{2}(2)$, it remains to bound the $L^{2}$-norm of $|x|^{2} w$. For this purpose, we write Equation (5.18) in another form:

$$
\begin{array}{r}
\partial_{t} w-\nu(I-\alpha \Delta)^{-1} \Delta w+(I-\alpha \Delta)^{-1}\left(u_{1} \cdot \nabla(w-\alpha \Delta w)\right) \\
+(I-\alpha \Delta)^{-1}\left(\left(u_{1}-u_{2}\right) \cdot \nabla\left(w_{2}-\alpha \Delta w_{2}\right)\right)=0
\end{array}
$$


Now, taking the scalar product of the above equation with $|x|^{4} w$, we get

$$
\begin{aligned}
\frac{1}{2} \partial_{t} \| \mid & \left.x\right|^{2} w \|_{L^{2}}^{2}-\nu\left((I-\alpha \Delta)^{-1} \Delta w,|x|^{4} w\right) \\
& +\left((I-\alpha \Delta)^{-1}\left(u_{1} \cdot \nabla(w-\alpha \Delta w)\right),|x|^{4} w\right) \\
& +\left((I-\alpha \Delta)^{-1}\left(\left(u_{1}-u_{2}\right) \cdot \nabla\left(w_{2}-\alpha \Delta w_{2}\right)\right),|x|^{4} w\right)=0
\end{aligned}
$$

We begin by computing the term $I_{1}=-\left((I-\alpha \Delta)^{-1} \Delta w,|x|^{4} w\right)$.

Let $z=(I-\alpha \Delta)^{-1} w$. Integrating by parts, we can write

$$
\begin{gathered}
I_{1}=-\int_{\mathbb{R}^{2}} \Delta z \cdot|x|^{4} z d x+\alpha \int_{\mathbb{R}^{2}} \Delta z \cdot|x|^{4} \Delta z d x \\
=\left\||x|^{2} \nabla z\right\|_{L^{2}}^{2}-8\||x| z\|_{L^{2}}^{2}+\alpha\left\||x|^{2} \Delta z\right\|_{L^{2}}^{2} \\
=\left\||x|^{2} \nabla(I-\alpha \Delta)^{-1} w\right\|_{L^{2}}^{2}-8\left\||x|(I-\alpha \Delta)^{-1} w\right\|_{L^{2}}^{2} \\
\quad+\alpha\left\||x|^{2} \Delta(I-\alpha \Delta)^{-1} w\right\|_{L^{2}}^{2}
\end{gathered}
$$

Next, we estimate the term $I_{2}=\left((I-\alpha \Delta)^{-1}\left(u_{1} \cdot \nabla(w-\alpha \Delta w)\right),|x|^{4} w\right)$. We have

$$
\begin{aligned}
& I_{2}=\int_{\mathbb{R}^{2}} u_{1} \cdot \nabla(I-\alpha \Delta)^{2} z \cdot(I-\alpha \Delta)^{-1}|x|^{4}(z-\alpha \Delta z) d x \\
& =\int_{\mathbb{R}^{2}} u_{1} \cdot \nabla(I-\alpha \Delta)^{2} z \cdot(I-\alpha \Delta)^{-1}\left[(I-\alpha \Delta)\left(|x|^{4} z\right)\right. \\
& \left.\quad \quad+\alpha \Delta\left(|x|^{4}\right) z+2 \alpha \nabla\left(|x|^{4}\right) \cdot \nabla z\right] d x \\
& \quad=\int_{\mathbb{R}^{2}} u_{1} \cdot \nabla(I-\alpha \Delta)^{2} z \cdot|x|^{4} z d x \\
& \quad \quad+\alpha \int_{\mathbb{R}^{2}}(I-\alpha \Delta)^{-1}\left(u_{1} \cdot \nabla(I-\alpha \Delta)^{2} z\right) \cdot\left(16|x|^{2} z+8|x|^{2} x \cdot \nabla z\right) d x
\end{aligned}
$$

Let

$$
\begin{aligned}
J_{1} & =\int_{\mathbb{R}^{2}} u_{1} \cdot \nabla(I-\alpha \Delta)^{2} z \cdot|x|^{4} z d x \\
& =\int_{\mathbb{R}^{2}}\left(u_{1} \cdot \nabla z-2 \alpha u_{1} \cdot \nabla \Delta z+\alpha^{2} u_{1} \cdot \nabla \Delta^{2} z\right) \cdot|x|^{4} z d x
\end{aligned}
$$

Integrating by parts and using (4.45) and Lemma 2.2 part a), we obtain

$$
\begin{aligned}
\left.\left|\int_{\mathbb{R}^{2}} u_{1} \cdot \nabla z\right| x\right|^{4} z d x \mid & =\left.\left|-2 \int_{\mathbb{R}^{2}} u_{1} \cdot x\right| x\right|^{2}|z|^{2} d x \mid \\
& \leq C\left\|u_{1}\right\|_{L^{\infty}}\|z\|_{L^{2}(2)}^{2} \leq C\|z\|_{L^{2}(2)}^{2}
\end{aligned}
$$

We also have

$$
\begin{aligned}
& \left.\left|\int_{\mathbb{R}^{2}} u_{1} \cdot \nabla \Delta z\right| x\right|^{4} z d x \mid \\
& \quad=\left.\left|-\int_{\mathbb{R}^{2}} u_{1} \cdot \nabla z\right| x\right|^{4} \Delta z d x-4 \int_{\mathbb{R}^{2}} u_{1} \cdot x \Delta z|x|^{2} z d x \mid \\
& \quad \leq C\left\|u_{1}\right\|_{L^{\infty}}\left\||x|^{2} \Delta z\right\|_{L^{2}}\left(\left\||x|^{2} \nabla z\right\|_{L^{2}}+\||x| z\|_{L^{2}}\right) \\
& \quad \leq C\left\||x|^{2} \Delta z\right\|_{L^{2}}\left(\left\||x|^{2} \nabla z\right\|_{L^{2}}+\|z\|_{L^{2}(2)}\right)
\end{aligned}
$$


Finally, integrating by parts several times, we get

$$
\begin{aligned}
& \int_{\mathbb{R}^{2}} u_{1} \cdot \nabla \Delta^{2} z \cdot|x|^{4} z d x=\int_{\mathbb{R}^{2}} \Delta u_{1} \cdot \nabla \Delta z \cdot|x|^{4} z d x \\
& \quad+\int_{\mathbb{R}^{2}} u_{1} \cdot \nabla \Delta z \cdot \Delta\left(|x|^{4} z\right) d x+2 \sum_{k} \int_{\mathbb{R}^{2}} \partial_{k} u_{1} \cdot \nabla \Delta z \cdot \partial_{k}\left(|x|^{4} z\right) d x \\
& =-\int_{\mathbb{R}^{2}} \Delta u_{1} \cdot \nabla\left(|x|^{4} z\right) \Delta z d x-\int_{\mathbb{R}^{2}} u_{1} \cdot \nabla \Delta\left(|x|^{4} z\right) \Delta z d x \\
& \quad-2 \sum_{k} \int_{\mathbb{R}^{2}} \partial_{k} u_{1} \cdot \nabla \partial_{k}\left(|x|^{4} z\right) \Delta z d x
\end{aligned}
$$

A simple computation and the application of the Hölder inequality allow us to write that

$$
\begin{aligned}
& \left.\left|\int_{\mathbb{R}^{2}} u_{1} \cdot \nabla \Delta^{2} z \cdot\right| x\right|^{4} z d x \mid \\
& \leq C\left\|\Delta u_{1}\right\|_{L^{4}}\left\||x|^{2} \Delta z\right\|_{L^{2}}\left(\left\||x|^{2} \nabla z\right\|_{L^{4}}+\||x| z\|_{L^{4}}\right) \\
& +C\left\|u_{1}\right\|_{L^{\infty}}\left[\|\Delta z\|_{L^{2}}\left(\||x| z\|_{L^{2}}+\left\||x|^{2} \nabla z\right\|_{L^{2}}\right)+\left\||x|^{2} \Delta z\right\|_{L^{2}}\||x| \Delta z\|_{L^{2}}\right] \\
& +C\left\|\nabla u_{1}\right\|_{L^{\infty}}\left\||x|^{2} \Delta z\right\|_{L^{2}}\left(\|z\|_{L^{2}}+\||x| \nabla z\|_{L^{2}}+\left\||x|^{2} \Delta z\right\|_{L^{2}}\right)
\end{aligned}
$$

Thus, inequalities (5.19) and (5.20) imply that

$$
\begin{aligned}
& \left.\left|\int_{\mathbb{R}^{2}} u_{1} \cdot \nabla \Delta^{2} z \cdot\right| x\right|^{4} z d x \mid \\
& \leq C\left\||x|^{2} \Delta z\right\|_{L^{2}}\left(\left\||x|^{2} \nabla z\right\|_{H^{1}}+\||x| z\|_{H^{1}}\right) \\
& \quad+C\left[\|\Delta z\|_{L^{2}}\left(\|z\|_{L^{2}(2)}+\left\||x|^{2} \nabla z\right\|_{L^{2}}\right)+\left\||x|^{2} \Delta z\right\|_{L^{2}}\|\Delta z\|_{L^{2}(2)}\right] \\
& \quad+C\left\||x|^{2} \Delta z\right\|_{L^{2}}\left(\|z\|_{L^{2}}+\|\nabla z\|_{L^{2}(2)}+\left\||x|^{2} \Delta z\right\|_{L^{2}}\right) \\
& \quad \leq C\left\||x|^{2} \Delta z\right\|_{L^{2}}\left(\|z\|_{L^{2}(2)}+\|\nabla z\|_{L^{2}(2)}+\|\Delta z\|_{L^{2}(2)}\right) \\
& \quad+C\|\Delta z\|_{L^{2}\left(\|z\|_{L^{2}(2)}+\left\||x|^{2} \nabla z\right\|_{L^{2}}\right)}
\end{aligned}
$$

Then, adding the inequalities (5.25), (5.26) and (5.27), we can write the following bound on $J_{1}$

$$
\begin{aligned}
& \left|J_{1}\right| \leq \\
& \quad C\|z\|_{L^{2}(2)}^{2}+\alpha C\left\||x|^{2} \Delta z\right\|_{L^{2}}\left(\|z\|_{L^{2}(2)}+(1+\alpha)\|\nabla z\|_{L^{2}(2)}+\alpha\|\Delta z\|_{L^{2}(2)}\right) \\
& \quad+\alpha^{2} C\|\Delta z\|_{L^{2}}\left(\|z\|_{L^{2}(2)}+\left\||x|^{2} \nabla z\right\|_{L^{2}}\right)
\end{aligned}
$$

Therefore, Lemma 5.1 implies that

$$
\left|J_{1}\right| \leq \tilde{C}_{\alpha}\|w\|_{L^{2}(2)}^{2},
$$

where $\tilde{C}_{\alpha}=C\left(1+\sqrt{\alpha}+\alpha+\frac{1+\alpha}{\sqrt{\alpha}}\right)$ and $C$ is a positive constant.

Now, let $J_{2}=\alpha \int_{\mathbb{R}^{2}}(I-\alpha \Delta)^{-1}\left(u_{1} \cdot \nabla(I-\alpha \Delta)^{2} z\right) \cdot\left(16|x|^{2} z+8|x|^{2} x . \nabla z\right) d x$. 
Integrating by parts and applying the Hölder inequality, we can write

$$
\begin{aligned}
& \left|J_{2}\right|=\alpha \mid \sum_{i} \int_{\mathbb{R}^{2}}(I-\alpha \Delta)^{-1}\left(u_{1 i}(I-\alpha \Delta)^{2} z\right) \cdot\left(32 x_{i} z+24|x|^{2} \partial_{i} z\right. \\
& \left.\quad+16 x_{i} x \cdot \nabla z+8|x|^{2} x \cdot \nabla \partial_{i} z\right) d x \mid \\
& \leq \alpha C\left(\||x| z\|_{L^{2}}+\left\||x|^{2} \nabla z\right\|_{L^{2}}\right) \sum_{i}\left\|(I-\alpha \Delta)^{-1}\left(u_{1 i}(I-\alpha \Delta)^{2} z\right)\right\|_{L^{2}} \\
& +\alpha C\left(\||x| \nabla z\|_{L^{2}}+\left\||x|^{2} \Delta z\right\|_{L^{2}}\right) \sum_{i}\left\||x|(I-\alpha \Delta)^{-1}\left(u_{1 i}(I-\alpha \Delta)^{2} z\right)\right\|_{L^{2}}
\end{aligned}
$$

Using Lemma 5.2 parts a), we can write

$$
\begin{aligned}
& \left\|(I-\alpha \Delta)^{-1}\left(u_{1 i}(I-\alpha \Delta)^{2} z\right)\right\|_{L^{2}} \\
& \quad \leq\left\|(I-\alpha \Delta)^{-1}\left(u_{1 i}(I-\alpha \Delta)^{2} z\right)-u_{1 i} w\right\|_{L^{2}}+\left\|u_{1 i} w\right\|_{L^{2}} \\
& \quad \leq C_{\alpha}\left(\left\|\Delta u_{1}\right\|_{L^{2}}\|w\|_{H^{1}}+\left\|\nabla u_{1}\right\|_{L^{4}}\|\nabla w\|_{L^{2}}\right)+C\left\|u_{1}\right\|_{L^{\infty}}\|w\|_{L^{2}} \\
& \quad \leq C_{\alpha}\|w\|_{H^{1}}
\end{aligned}
$$

On the other hand, Lemma 5.2 parts b) allows us to write

$$
\begin{aligned}
& \left\||x|(I-\alpha \Delta)^{-1}\left(u_{1 i}(I-\alpha \Delta)^{2} z\right)\right\|_{L^{2}} \\
& \leq\left\||x|\left[(I-\alpha \Delta)^{-1}\left(u_{1 i}(I-\alpha \Delta)^{2} z\right)-u_{1 i} w\right]\right\|_{L^{2}}+\left\||x| u_{1 i} w\right\|_{L^{2}} \\
& \leq C_{\alpha}\|w\|_{H^{1}}+C_{\alpha}\left(\left\|\Delta u_{1}\right\|_{L^{4}}\||x| w\|_{L^{2}}+\left\|\nabla u_{1}\right\|_{L^{\infty}}\|w\|_{L^{2}(2)}\right) \\
& +C\left\|u_{1}\right\|_{L^{\infty}}\||x| w\|_{L^{2}} \\
& \leq C_{\alpha}\left(\|w\|_{H^{1}}+\|w\|_{L^{2}(2)}\right)
\end{aligned}
$$

Thus, Inequalities (5.29) and (5.30), together with Lemma 5.1 imply that

$$
\left|J_{2}\right| \leq C_{\alpha}\left(\|w\|_{L^{2}(2)}\|w\|_{H^{1}}+\|w\|_{L^{2}(2)}^{2}\right)
$$

Finally, we estimate the term

$$
I_{3}=\left((I-\alpha \Delta)^{-1}\left(\left(u_{1}-u_{2}\right) \cdot \nabla\left(w_{2}-\alpha \Delta w_{2}\right)\right),|x|^{4} w\right) .
$$

Applying the Hölder inequality and using Lemma 5.2 parts c) and d), we can write

$$
\begin{aligned}
\left|I_{3}\right| & \leq\left\||x|^{2}(I-\alpha \Delta)^{-1}\left(\left(u_{1}-u_{2}\right) . \nabla\left(w_{2}-\alpha \Delta w_{2}\right)\right)\right\|_{L^{2}}\left\||x|^{2} w\right\|_{L^{2}} \\
& \leq C_{\alpha}\left\|u_{1}-u_{2}\right\|_{L^{\infty}}\left\||x|^{2} w\right\|_{L^{2}}\left(\left\|\nabla w_{2}\right\|_{L^{2}(2)}+\left\|\Delta w_{2}\right\|_{L^{2}(2)}\right) \\
& \leq C_{\alpha}\|w\|_{L^{2}(2)}^{\frac{1}{2}}\|w\|_{H^{1}}^{\frac{1}{2}}\left\||x|^{2} w\right\|_{L^{2}}
\end{aligned}
$$

Therefore, collecting the inequalities (5.24), (5.28), (5.31) and (5.32), we can write Equality (5.23) as follows

$$
\begin{aligned}
& \frac{1}{2} \partial_{t}\left\||x|^{2} w\right\|_{L^{2}}^{2}+\nu\left\||x|^{2} \nabla(I-\alpha \Delta)^{-1} w\right\|_{L^{2}}^{2}+\alpha \nu\left\||x|^{2} \Delta(I-\alpha \Delta)^{-1} w\right\|_{L^{2}}^{2} \\
& \leq C_{\alpha}\left(\|w\|_{L^{2}(2)}\|w\|_{H^{1}}+\|w\|_{L^{2}(2)}^{2}\right)+C_{\alpha}\|w\|_{L^{2}(2)}^{\frac{1}{2}}\|w\|_{H^{1}}^{\frac{1}{2}}\left\||x|^{2} w\right\|_{L^{2}}
\end{aligned}
$$


Taking the sum of Inequalities (5.21) and (5.33) and applying the Cauchy-Schwarz inequality, we obtain

$$
\begin{aligned}
& \frac{1}{2} \partial_{t}\left(\|w\|_{L^{2}(2)}^{2}+\alpha\|\nabla w\|_{L^{2}}^{2}\right)+\nu\|\nabla w\|_{L^{2}}^{2}+\nu\left\||x|^{2} \nabla(I-\alpha \Delta)^{-1} w\right\|_{L^{2}}^{2} \\
& \left.+\alpha \nu\left\||x|^{2} \Delta(I-\alpha \Delta)^{-1} w\right\|_{L^{2}}^{2} \leq\left(C_{\alpha}+\tilde{C}_{\alpha}\right)\|w\|_{L^{2}(2)}^{2}+C_{\alpha}\|w\|_{H^{1}}^{2}\right)
\end{aligned}
$$

Finally, integrating the above inequality between 0 and $t>0$ and using the Gronwall lemma, we deduce that $w=0$ and thus, the solution of Equation (1.1) is unique.

5.3. Proof of Corollary 1.2. In the proof of Corollary 1.2, we use the proposition B.1 of [16], that we recall here.

Proposition 5.3. Let $w \in L^{2}\left(m^{\star}\right)$ for some $m^{\star}>0$ and denote by $v$ the velocity field obtained from $w$ via the Biot-Savart law. Assume that either 1) $0<$ $m^{\star} \leq 1$, or

2) $1<m^{\star} \leq 2$, and $\int_{\mathbb{R}^{2}} w(\xi) d \xi=0$

If $m^{\star} \notin \mathbb{N}$, then for all $2<q<\infty$, there exists $C>0$ such that

$$
\left\|\left(1+|\xi|^{2}\right)^{\frac{m^{\star}}{2}-\frac{1}{q}} v\right\|_{L^{q}} \leq C\left\|\left(1+|\xi|^{2}\right)^{\frac{m^{\star}}{2}} w\right\|_{L^{2}} .
$$

For the proof of the above proposition, see [16].

Now, let $w(x, t)$ be the solution of (1.1) with $w(x, 0)=w_{0}(x)$ and let $W(\xi, \tau)$ be the solution of (1.6) with $W_{0}(\xi)=T w_{0}(x)$ as initial data. We recall that $L^{2}(2)$ is continuously embedded into $L^{p}\left(\mathbb{R}^{2}\right)$, for all $1 \leq p \leq 2$.

Then, using (1.3) and Theorem 1.1, we obtain

$$
\begin{aligned}
\left\|\left(1-\frac{\alpha}{T} \Delta\right)(w(t)-\beta \Omega(t))\right\|_{L^{p}} \\
\quad=\nu^{\frac{1}{p}}(T+t)^{-1+\frac{1}{p}}\left\|\left(1-\bar{\alpha} e^{-\tau} \Delta\right)(W(\log (T+t))-\beta G)\right\|_{L^{p}} \\
\quad \leq C(T+t)^{-1+\frac{1}{p}}\left\|\left(1-\bar{\alpha} e^{-\tau} \Delta\right)(W(\log (T+t))-\beta G)\right\|_{L^{2}(2)} \\
\quad \leq C(T+t)^{-1-\theta+\frac{1}{p}}
\end{aligned}
$$

Using Lemma 2.1 part a), we see that (1.13) holds for all $2<q<\infty$.

In order to show that (1.13) holds also for all $1<q \leq 2$, we use Proposition 5.3.

Now, assume that $1<q \leq 2$ and fix $m^{\star} \in\left(\frac{2}{q}, 2\right)$.

Therefore, using Proposition 5.3 and the Hölder inequality, we obtain

$$
\begin{aligned}
\left\|\left(1-\bar{\alpha} e^{-\tau} \Delta\right)\left(V(\tau)-\beta V^{G}(\tau)\right)\right\|_{L^{q}} \\
\leq\left\|\left(1+|\xi|^{2}\right)^{\frac{m}{2}-\frac{1}{4}}\left(1-\bar{\alpha} e^{-\tau} \Delta\right)\left(V(\tau)-\beta V^{G}(\tau)\right)\right\|_{L^{4}} \\
\leq C\left\|\left(1+|\xi|^{2}\right)^{\frac{m}{2}}\left(1-\bar{\alpha} e^{-\tau} \Delta\right)(W(\tau)-\beta G(\tau))\right\|_{L^{2}} \\
\leq\left\|\left(1-\bar{\alpha} e^{-\tau}\right)(W(\tau)-\beta G(\tau))\right\|_{L^{2}(2)} \leq C e^{-\theta \tau}
\end{aligned}
$$

Finally, using the change of variables (1.4), we obtain (1.13) for all $1<q \leq 2$. 


\section{References}

[1] A.Busuioc: Sur quelques problèmes en mécanique des fluides non newtoniens, Université de Paris 6, 2000

[2] A.Carpio: Asymptotic behavior for the vorticity equations in dimensions two and three, Comm. Partial Differential Equations, 1994, volume 19(5-6), pages 827-872

[3] A.Carpio: Large-time behavior in incompressible Navier-Stokes equations, SIAM J. Math. Anal., 1996, volume 27(2), pages 449-475

[4] Th.Cazenave and A.Haraux: Introduction aux problèmes d'évolution semi-linéaires, Math. Appl.1, Ellipses, Paris, 1990

[5] D.Cioranescu et V.Girault: Weak and classical solutions of a family of second grade fluids, Int. J. Nonlinear Mechanics, 1997, volume 32, pages 317-335

[6] D.Cioranescu and E.H.Ouazar: Existence and uniqueness for fluids of second grade, Nonlinear Partial Differential Equations, Collège de France Seminar Pitman, 1984, volume 109, pages $178-197$

[7] J.E.Dunn and R.L.Fosdick: Thermodynamics, stability and boundedness of fluids of complexity two and fluids of second grade, Arch. Rat. Mech. Anal., 1974, volume 3, 56, pages 191-252

[8] R.L.Fosdick and K.R.Rajakopal: Anomalous features in the model of second order fluids, Arch. Rat. Mech. Anal., 1979, volume 3, 70, pages 1-46

[9] R.L.Fosdick and K.R.Rajakopal: Thermodynamics and stability of fluids of and third grade, Proc. Royal Soc. London, 1980, volume A 339, pages 351-377

[10] G.P.Galdi: Mathemathical theory of second-grade fluids, Stability and Wave propagation in Fluids and Solids, CISM Course and Lectures, Springer-Verlag, New York, 1995, volume 344, pages $67-104$

[11] G.P.Galdi, M.Grobbelaar-Van Dalsen and N.Sauer: Existence and uniqueness of classical solutions of the equations of motion for second-grade fluids, Arch. Rational Mech. Anal., 1993, volume 124, pages 221-237

[12] G.P.Galdi and A.Sequeira: Further existence results for classical solutions of the equations of a second-grade fluid, Arch. Rational Mech. Anal., 1994, volume 128, pages 297-312

[13] Th.Gallay and G.Raugel: Scaling variables and asymptotic expansions in damped wave equations, J. Diff. Eq., 1998, volume 150, pages 42-97

[14] Th.Gallay and G.Raugel: Scaling variables and stability of hyperbolic fronts, SIAM J. Math. Anal., 2000, volume 32, pages 1-29

[15] Th.Gallay and L. Miguel Rodrigues : Sur le temps de vie de la turbulence bidimensionnelle, Annales de la Faculté des Sciences de Toulouse, 2008, Sér. 6, volume 17, no. 4, pages 719-733

[16] Th.Gallay and C.Eugene Wayne: Invariant manifolds and the long-time asymptotics of the Navier-Stokes and vorticity equations on $\mathbb{R}^{2}$, Arch. Ration. Mech. Anal., 2002, volume 163(3), pages $209-258$

[17] Th.Gallay and C.Eugene Wayne: Global stability of vortex solutions of the two-dimensional Navier-Stokes equation, Comm. Math. Phys., 2005, volume 255, pages 97-129

[18] D.Iftimie: Remarques sur la limite $\alpha \rightarrow 0$ pour les fluides de grade deux, Comm. Math. Phys., 2005, volume 255, pages 97-129

[19] T.Kato: Strong $L^{p}$ - solutions of the Navier-Stokes equation in $\mathbb{R}^{m}$, with applications to weak solutions, Math. Z., 1984, volume 187(4), pages 471-480

[20] I.Moise, R.Rosa and X.Wang: Attractors for non-compact semigroups via energy equations, Nonlinearity, 1998, volume 11, pages 1369-1393

[21] M.Paicu, G.Raugel and A.Rekalo: Regularity of the global attractor and finite-dimensional behavior for the second grade fluid equations, 2008,

[22] A.Pazy: Semi groups of linear operators and applications to partial differential equations, Appl. Math. Sci 44. Springer. New-York, 1983

[23] R.S.Rivlin and Ericksen : Stress-deformation relations for isotropic materials, J. Rational Mech. Anal., 1955, volume 4, pages 323-425

[24] L. Miguel Rodrigues : Asymptotic stability of Oseen vortices for a density-dependent incompressible viscous fluid, Annales de l'I.H.P. (C) - Analyse non linaire, 2009, volume 26, pages 625-648

[25] M.Wiegner: Decay results for weak solutions of the Navier-Stokes equations on $\mathbb{R}^{n}$, J. Lond. Math. Soc., II. Ser., 1987, volume 35, pages 303-313 
Laboratoire de Mathématiques, Université Paris-Sud, Bat. 425, 91405 Orsay Cedex, FRANCE

E-mail address: basma.jaffal@math.u-psud.fr 